\title{
Ensemble generalized Kohn-Sham theory: The good, the bad, and the ugly ${ }^{\circledR}$
}

Cite as: J. Chem. Phys. 154, 094125 (2021); https://doi.org/10.1063/5.0040447

Submitted: 13 December 2020 . Accepted: 11 February 2021 . Published Online: 05 March 2021

\begin{abstract}
(i) Tim Gould, and (D) Leeor Kronik
COLLECTIONS

EP This paper was selected as an Editor's Pick
\end{abstract}
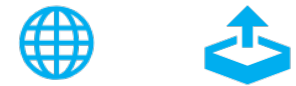

\section{ARTICLES YOU MAY BE INTERESTED IN}

Configuration interaction trained by neural networks: Application to model polyaromatic hydrocarbons

The Journal of Chemical Physics 154, 094117 (2021); https://doi.org/10.1063/5.0040785

$r^{2}$ SCAN-D4: Dispersion corrected meta-generalized gradient approximation for general chemical applications

The Journal of Chemical Physics 154, 061101 (2021); https://doi.org/10.1063/5.0041008

$r^{2}$ SCAN-3C: A "Swiss army knife" composite electronic-structure method

The Journal of Chemical Physics 154, 064103 (2021); https://doi.org/10.1063/5.0040021

\section{Challenge us.}

What are your needs for periodic signal detection?

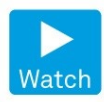

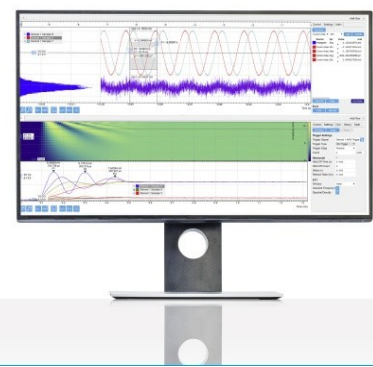

Zurich

Instruments 


\title{
Ensemble generalized Kohn-Sham theory: The good, the bad, and the ugly
}

\author{
Cite as: J. Chem. Phys. 154, 094125 (2021); doi: 10.1063/5.0040447 \\ Submitted: 13 December 2020 - Accepted: 11 February 2021 • \\ Published Online: 5 March 2021
}

\author{
Tim Gould ${ }^{1, a)}$ (D) and Leeor Kronik ${ }^{2, a)}$ (D)
}

\author{
AFFILIATIONS \\ ${ }^{1}$ QLD Micro- and Nanotechnology Centre, Griffith University, Nathan, QLD 4111, Australia \\ ${ }^{2}$ Department of Materials and Interfaces, Weizmann Institute of Science, Rehovoth 76100, Israel
}

a) Authors to whom correspondence should be addressed: t.gould@griffith.edu.au and leeor.kronik@weizmann.ac.il

\begin{abstract}
Two important extensions of Kohn-Sham (KS) theory are generalized: KS theory and ensemble KS theory. The former allows for nonmultiplicative potential operators and greatly facilitates practical calculations with advanced, orbital-dependent functionals. The latter allows for quantum ensembles and enables the treatment of open systems and excited states. Here, we combine the two extensions, both formally and practically, first via an exact yet complicated formalism and then via a computationally tractable variant that involves a controlled approximation of ensemble "ghost interactions" by means of an iterative algorithm. The resulting formalism is illustrated using selected examples. This opens the door to the application of generalized KS theory in more challenging quantum scenarios and to the improvement of ensemble theories for the purpose of practical and accurate calculations.
\end{abstract}

Published under license by AIP Publishing. https://doi.org/10.1063/5.0040447

\section{INTRODUCTION}

Kohn-Sham ${ }^{1,2}$ (KS) density functional theory (DFT) has proven to be an indispensable tool for the first principles calculations across a wide range of disciplines. ${ }^{3,4}$ Many widely used density functional approximations (DFAs), e.g., Refs. 5-10, are "hybrid functionals," i.e., they mix exact (Fock) exchange with KS exchange. These are almost always applied using the non-multiplicative Fockexchange operator, which places them outside the realm of KS theory, but well within generalized Kohn-Sham (GKS) theory. ${ }^{11-14}$ The popularity of hybrid functionals reflects three useful properties of hybrids: first, the inclusion of exact Fermionic exchange allows for a systematically higher predictive accuracy; ${ }^{15}$ second, this is carried out in a numerically straightforward fashion, whereas the use of Fock exchange within "pure" KS theory must rely on difficult to calculate optimized effective potentials (OEPs); ${ }^{16-21}$ and third, the non-multiplicative potential allows for a reduction and, in some circumstances, even the elimination of the KS derivative discontinuity, ${ }^{22}$ allowing one to overcome notoriously difficult problems for KS theory such as the bandgap problem ${ }^{23,24}$ or the charge transfer excitation problem.
Recent years have seen renewed interest in ensemble KS (EKS) theory, which broadens the scope of traditional KS theory to include mapping to an ensemble of reference non-interacting electron states. EKS, thereby, extends the reach of DFT to a more diverse range of quantum scenarios, e.g., systems with non-integer electron number $^{22,28-34}$ and mixtures of ground- and low-lying excited states. ${ }^{35-3}$ It can also improve the treatment of quantum systems that are technically within the reach of KS theory, such as those with significant spin-contamination. ${ }^{3}$

Ensemble DFT (EDFT) is especially useful for addressing several classes of problems that are inaccessible to standard DFT. First, excited-state EDFT $^{28,33,35-59}$ has attracted significant recent attention as a route to low cost prediction of charge transfer $^{40,57}$ and double ${ }^{40,51,54}$ excitations, which are difficult to predict using traditional time-dependent KS theory. Second, approximations based on excited state EDFT have been introduced and employed to solve strong correlation problems in various complex chemical systems-see, e.g., Filatov's review in $2015^{41}$ of such approaches. Third, ensemble theory of fractional ions ${ }^{22}$ and " $N$ cenetred"33 mixtures offer direct access to fundamental and optical gaps at the Kohn-Sham level. ${ }^{29,30,33,34}$ Finally, the thermal 
generalization of $\mathrm{EDFT}^{61}$ allows quantum systems to be studied at finite temperature, albeit with additional complexities compared to other applications of ensemble DFT. ${ }^{22,62}$

To date, we are not aware of any formal theory that has been able to combine rigorously the attractive features of GKS and EKS theories, though some ad hoc solutions ${ }^{39,41}$ and approximations ${ }^{55,60}$ have been reported. This hampers the application of ensemble theories, which must be solved non-self-consistently or with less-sophisticated approximations or using OEPs.

Here, we demonstrate how to combine EKS and GKS theories into an ensemble GKS (EGKS) theory. We show, however, that the resulting approach is ill-suited to existing GKS implementations in its direct form. We then introduce simple, formally motivated approximations that make EGKS tractable without losing its good features.

\section{BACKGROUND THEORY}

\section{A. Ensemble Kohn-Sham theory}

We first briefly introduce EKS theory before turning to GKS and EGKS theory. For simplicity, we use a spin-unpolarized formalism throughout. To begin, we introduce the universal "Levy" ensemble density functional,

$$
\mathcal{F}^{\lambda}[n, \mathbf{w}]=\min _{\hat{\Gamma}_{\mathbf{w}} \rightarrow n} \operatorname{Tr}\left[(\hat{T}+\lambda \hat{W}) \hat{\Gamma}_{\mathbf{w}}\right],
$$

where $\lambda \in[0,1]$ is an adjustable interacting-strength parameter. Here, $n$ is the density, $\mathbf{w}$ is a set of statistical weights that define the ensemble, $\hat{T}$ is the kinetic energy operator, and $\hat{W}$ is the electronelectron interaction energy operator. The ensemble density matrix, $\hat{\Gamma}_{\mathrm{w}}=\sum_{\kappa} w_{\kappa}|\kappa\rangle\langle\kappa|$, involves a weighted sum over orthonormal wave functions $|\kappa\rangle . \hat{\Gamma}_{\mathbf{w}} \rightarrow n$ is short-hand for $\operatorname{Tr}\left[\hat{n}(\boldsymbol{r}) \hat{\Gamma}_{\mathbf{w}}\right]=n(\boldsymbol{r})$, where $\hat{n}(\boldsymbol{r})$ is the electron-density operator. By varying the weights, $\mathbf{w}$, we can obtain useful properties (e.g., excitation energies) of the system that are inaccessible to ground-state DFT.

The Levy functional $\mathcal{F}^{\lambda}$ provides a versatile framework for defining key functionals in EKS, ${ }^{53,56,58}$ specifically the KS kinetic energy $\left(\mathcal{T}_{s}\right)$, Hartree-exchange $(\mathrm{Hx})$, and correlation (c) functionals, respectively:

$$
\begin{gathered}
\mathcal{T}_{s}[n, \mathbf{w}]=\mathcal{F}^{0}[n, \mathbf{w}], \\
\mathcal{E}_{\mathrm{Hx}}[n, \mathbf{w}]=\lim _{\lambda \rightarrow 0^{+}} \frac{\mathcal{F}^{\lambda}[n, \mathbf{w}]-\mathcal{T}_{s}[n, \mathbf{w}]}{\lambda}, \\
\mathcal{E}_{\mathrm{c}}[n, \mathbf{w}]=\mathcal{F}^{1}[n, \mathbf{w}]-\mathcal{T}_{s}[n, \mathbf{w}]-\mathcal{E}_{\mathrm{Hx}}[n, \mathbf{w}] .
\end{gathered}
$$

Each is a functional of the electron density $n$ and weights $\mathbf{w}$, denoted by $[n, \mathbf{w}]$.

It has recently been shown that $\mathcal{T}_{s}[n, \mathbf{w}] \equiv \operatorname{Tr}\left[\hat{\Gamma}_{s, \mathbf{w}} \hat{T}\right]$ and $\mathcal{E}_{\mathrm{Hx}}[n, \mathbf{w}] \equiv \operatorname{Tr}\left[\hat{\Gamma}_{s, \mathbf{w}} \hat{W}\right],{ }^{56}$ for some non-interacting ensemble density matrix $\hat{\Gamma}_{s, \mathbf{w}}$, which is formed on configuration state functions. $\mathcal{T}_{s}$ and $\mathcal{E}_{\mathrm{Hx}}$ are, thereby, functionals of a set of orbitals, $\{\phi\}$,

$$
\begin{aligned}
& \mathcal{T}_{s}[\{\phi\}]=\sum_{i} f_{i}^{\mathbf{w}} \int d \boldsymbol{r} \phi_{i}^{*}(\boldsymbol{r}) \hat{t} \phi_{i}(\boldsymbol{r}) \equiv \sum_{i} f_{i}^{\mathbf{w}} t_{i, \phi}, \\
& \mathcal{E}_{\mathrm{Hx}}[\{\phi\}]=\frac{1}{2} \sum_{i j}\left\{F_{i j}^{J, \mathbf{w}}(i j \mid i j)_{\phi}^{W}+F_{i j}^{K, \mathbf{w}}(i j \mid j i)_{\phi}^{W}\right\},
\end{aligned}
$$

involving electron-repulsion integrals $(i j \mid k l)_{\phi}^{W}=\int d \boldsymbol{r} d \boldsymbol{r}^{\prime} W\left(\boldsymbol{r}, \boldsymbol{r}^{\prime}\right)$ $\phi_{i}^{*}(\boldsymbol{r}) \phi_{j}\left(\boldsymbol{r}^{\prime}\right) \phi_{k}(\boldsymbol{r}) \phi_{l}^{*}\left(\boldsymbol{r}^{\prime}\right)$ with $W=\frac{1}{\left|\boldsymbol{r}-\boldsymbol{r}^{\prime}\right|} \cdot{ }^{63}$ The coefficients $f_{i}^{\mathbf{w}} \in$ $[0,2]$ are average occupancies of orbitals $i$ across the ensemble. The pair-coefficients, $F_{i j}^{J, \mathbf{w}}$ and $F_{i j}^{K, \mathbf{w}}$, encode all information about the non-interacting ensemble and are ensemble-specific. They are more complicated to obtain than $f_{i}$; some examples are provided in the Appendix. Importantly, these pair-coefficients are not necessarily (scaled) products of $f_{i}^{\mathrm{w}}$, i.e., generally, $F_{i j}^{J} \neq-2 F_{i j}^{K} \neq f_{j} f_{j}$. Recent work has used the fluctuation-dissipation theorem to show that separated $\mathcal{E}_{\mathrm{H}}$ and $\mathcal{E}_{\mathrm{x}}$ also yield functionals of similar form to Eq. (6). For pedagogical simplicity, we shall not consider the separated terms further.

Just as in KS theory, the EKS orbitals, $\phi_{i}[n, \mathbf{w}](\boldsymbol{r})$, are eigensolutions of the KS equation,

$$
\left\{\hat{t}+v+v_{\mathrm{Hxc}}[n, \mathbf{w}]\right\} \phi_{i}[n, \mathbf{w}]=\epsilon_{i}[n, \mathbf{w}] \phi_{i}[n, \mathbf{w}] .
$$

Here, $v_{\mathrm{Hxc}}[n, \mathbf{w}]=\frac{\delta \mathcal{E}_{\mathrm{Hx}}[n, \mathbf{w}]}{\delta n}+\frac{\delta \mathcal{E}_{\mathrm{c}}[n, \mathbf{w}]}{\delta n}$ is the multiplicative Hxc effective potential of EKS theory, and $v$ is the external potential. ${ }^{36}$ The effective potential, $v_{s}:=v+v_{\mathrm{Hxc}}$, applied to the electrons, thus depends both on the overall ensemble density, $n$, and the set of weights, w.

\section{B. Generalized Kohn-Sham theory}

We now turn to GKS theory. Seidl et al. ${ }^{11}$ rigorously showed that the usual KS equation can be modified to include a non-local operator $\hat{v}_{S}$, which is usually of Fock-like form, without changing the fundamental properties of density functionals. The generalized KS equation,

$$
\left[\hat{t}+v+\hat{v}_{S}+v_{R}[n]\right] \varphi_{i}[n]=\varepsilon_{i}[n] \varphi_{i}[n]
$$

where $v$ is the external potential and $v_{R}$ is a multiplicative effective potential, can then be used to find orbitals $\{\varphi\}$ and the density $n$. We obtain different operators $\hat{v}_{S}$ and potentials $v_{R}$ for different orbital functionals $S[\{\varphi\}]$ that are invariant to unitary transformations of the orbitals. We use $\{\varphi\}$ to indicate orbital solutions of Eq. (8), as opposed to $\{\phi\}$ of Eq. (7).

The existence of the GKS equation is deduced by defining $S[n]=\min _{\{\varphi\} \rightarrow n} S[\{\varphi\}]$ and $R_{S}[n]=F^{1}[n]-S[n]$, where we use non-calligraphic $F^{1}$ to represent the special case of Eq. (1) for pure ground states $\left(w_{0}=1, w_{\kappa \neq 0}=0\right)$. Then, the ground-state energy, $E_{0}=\min _{n}\left\{S[n]+R_{S}[n]+V[n]\right\}=\min _{\{\varphi\}}\left\{S[\{\varphi\}]+R_{S}\left[n_{\varphi}\right]\right.$ $\left.+V\left[n_{\varphi}\right]\right\}$, is minimized for the density $n \equiv n_{\varphi} \equiv n_{0}$. Uniqueness (in non-degenerate systems) follows from the external potential $v$ being uniquely defined by the ground state density $n_{0}$. Here, $V[n]$ $=\int d \boldsymbol{r} n(\boldsymbol{r}) v(\boldsymbol{r})$, and we used $S+R_{S}=F^{1}$.

Importantly, almost all practical hybrid approximations can be re-expressed as functionals of the first-order reduced density matrix (1-RDM), $\gamma_{1}\left(\boldsymbol{r}, \boldsymbol{r}^{\prime}\right)=\sum_{i \in \mathrm{occ}} \varphi_{i}^{*}(\boldsymbol{r}) \varphi_{i}\left(\boldsymbol{r}^{\prime}\right)$. This includes all Hartree-Fock-like expressions, $S[\{\varphi\}]:=\left\langle\Phi\left|\hat{T}+\hat{W}_{S}\right| \Phi\right\rangle$, where $\Phi$ is a Slater determinant formed from $\{\varphi\}$ and $\hat{W}_{S}$ is a (modified) interaction term involving interactions of form $0<W_{S}\left(\left|\boldsymbol{r}-\boldsymbol{r}^{\prime}\right|\right) \leq W\left(\left|\boldsymbol{r}-\boldsymbol{r}^{\prime}\right|\right)$, which can be a simple fraction 
$W_{S}=\alpha W$ of the full interaction term, or a more complex rangeseparated expression. ${ }^{8,64,65}$ Then,

$$
\begin{aligned}
S\left[\gamma_{1}\right] \equiv & \int-\left.\frac{1}{2} \nabla^{2} \gamma_{1}\left(\boldsymbol{r}, \boldsymbol{r}^{\prime}\right)\right|_{\boldsymbol{r}=\boldsymbol{r}^{\prime}} d \boldsymbol{r} \\
& +\int W_{S}\left(\boldsymbol{r}, \boldsymbol{r}^{\prime}\right)\left[n(\boldsymbol{r}) n\left(\boldsymbol{r}^{\prime}\right)-\frac{1}{2}\left|\gamma_{1}\left(\boldsymbol{r}, \boldsymbol{r}^{\prime}\right)\right|^{2}\right] \frac{d \boldsymbol{r} d \boldsymbol{r}^{\prime}}{2} .
\end{aligned}
$$

We restrict ourselves to this broad and popular class of hybrids, though many results derived below are general.

Our first step toward EGKS theory is to recognize that Eq. (9) can also accept more general 1-RDMs,

$$
\gamma_{1}\left(\boldsymbol{r}, \boldsymbol{r}^{\prime}\right)=\sum_{i} f_{i} \varphi_{i}^{*}(\boldsymbol{r}) \varphi_{i}\left(\boldsymbol{r}^{\prime}\right),
$$

in which $f_{i} \in[0,2]$ can be non-integer [note $n(\boldsymbol{r})=\gamma_{1}(\boldsymbol{r}, \boldsymbol{r})$ ]. Such 1-RDMs appear naturally in ensemble theories, e.g., the 1$\mathrm{RDM}$ for a lithium cation with a partial charge $+q$ is $\gamma_{1}\left(\boldsymbol{r}, \boldsymbol{r}^{\prime}\right)$ $=2 \varphi_{1 s}^{*}(\boldsymbol{r}) \varphi_{1 s}\left(\boldsymbol{r}^{\prime}\right)+(1-q) \varphi_{2 s}^{*}(\boldsymbol{r}) \varphi_{2 s}\left(\boldsymbol{r}^{\prime}\right)$. Importantly, Eq. (9) can be obtained by the sum of Eqs. (5) and (6), for the special case of the product form $F_{i j}^{J}=-2 F_{i j}^{K}=f_{i} f_{j}$. Conveniently, the self-consistent orbitals from Eqs. (9) and (10) always obey Eq. (8). This is because the functional chain rule ensures that orbital derivatives, $\frac{\delta S[\{\varphi\}]}{\delta \varphi_{i}^{*}}$ $=\frac{\delta S\left[\gamma_{1}\right]}{\delta \gamma_{1}} \star \frac{\delta \gamma_{1}}{\delta \varphi_{i}^{*}}=f_{i}\left(\hat{t}+\hat{v}_{S}\right) \varphi_{i}$, attain the same form for all $i$. Here, $\hat{v}_{S} \varphi:=\frac{\delta\left(S-T_{s}\right)}{\delta \gamma_{1}} \star \varphi$, where $\star$ indicates any necessary integrals. Similarly, $\frac{\delta R_{S}}{\delta \varphi^{*}}:=\frac{\delta E_{R}}{\delta n} \frac{\delta n}{\delta \varphi_{i}^{*}} \equiv f_{i} v_{R} \varphi_{i}$, and normalization leads to $\varepsilon_{i} f_{i} \varphi_{i}$ because the resulting operator equation is Hermitian. Division by $f_{i}$ yields Eq. (8).

\section{ENSEMBLE GENERALIZED KOHN-SHAM THEORY}

\section{A. Fundamental theory}

We are now ready to tackle EGKS. The properly ensemblized version of Eq. (9) is $\mathcal{S}^{e}[\{\varphi\}, \mathbf{w}]:=\mathcal{T}_{s}[\{\varphi\}, \mathbf{w}]+\mathcal{E}_{\mathrm{Hx}, S}[\{\varphi\}, \mathbf{w}]$, which uses the ensemble functionals given in Eqs. (5) and (6) [but with $W \rightarrow W_{S}$ in (6)]. We define the ensemble density functionals,

$$
\begin{gathered}
\mathcal{S}^{e}[n, \mathbf{w}]:=\min _{\{\varphi\} \rightarrow n} \mathcal{S}^{e}[\{\varphi\}, \mathbf{w}], \\
\mathcal{R}_{S}^{e}[n, \mathbf{w}]:=\mathcal{F}^{1}[n, \mathbf{w}]-\mathcal{S}^{e}[n, \mathbf{w}],
\end{gathered}
$$

where $\{\varphi\} \rightarrow n$ is short-hand for $\sum_{i} f_{i}|\varphi(\boldsymbol{r})|^{2}=n(\boldsymbol{r})$. It is straightforward to show that Eqs. (11) and (12) are well-defined functionals: both are unique, bounded below $\left(\mathcal{T}_{s}[n] \leq \mathcal{S}^{e}[n], \mathcal{F}^{1}[n]-\mathcal{T}_{s}[n]\right.$ $\left.-\mathcal{E}_{\mathrm{Hx}, S}[n] \leq \mathcal{R}_{S}^{e}[n]\right)$, and have, at least, one valid solution $(\{\phi\}=\{\varphi\})$.

Equation (11) is maximally free ${ }^{56}$ of "ghost-interactions" (GI), namely, spurious interactions between electrons and their counterparts in different ensemble members ${ }^{66}$ that are analogous to selfinteractions in pure states. In other words, Eq. (11) will always yield the lowest possible energy for states that are consistent with the weights, w. In contrast, $S\left[\gamma_{1}^{\mathbf{w}}\right]$ of (9) generally exhibits GI errors. ${ }^{66}$ Therefore, it has intrinsic positive energy errors even at the exact Hartree-exchange level and is inconsistent with Eq. (6).

We therefore define $\mathcal{S}^{\mathrm{GI}}[\{\varphi\}, \mathbf{w}]:=\mathcal{S}^{e}[\{\varphi\}, \mathbf{w}]-S\left[\gamma_{1}^{\mathbf{w}}\right]$ $\equiv \mathcal{E}_{\mathrm{Hx}}[\{\varphi\}, \mathbf{w}]-E_{\mathrm{Hx}}\left[\gamma_{1}^{\mathbf{w}}\right]<0$, or equivalently,

$$
\begin{aligned}
\mathcal{S}^{\mathrm{GI}}[\{\varphi\}, \mathbf{w}]:= & \frac{1}{2} \sum_{i j}\left[\left(F_{i j}^{J, \mathbf{w}}-f_{i}^{\mathbf{w}} f_{j}^{\mathbf{w}}\right)(i j \mid i j)_{\varphi}^{W_{S}}\right. \\
& \left.+\left(F_{i j}^{K, \mathbf{w}}+\frac{1}{2} f_{i}^{\mathbf{w}} f_{j}^{\mathbf{w}}\right)(i j \mid j i)_{\varphi}^{W_{S}}\right] .
\end{aligned}
$$

This energy term accounts for differences between the GI-free orbital functional [Eq. (11)] and the 1-RDM energy functional [Eqs. (9) and (10)]. It is worth noting that Eq. (13) typically only has a small number of non-zero terms, as $F_{i j}^{J, \mathbf{w}}=f_{i}^{\mathbf{w}} f_{j}^{\mathbf{w}}$ and $F_{i j}^{K, \mathbf{w}}=-\frac{1}{2} f_{i}^{\mathbf{w}} f_{j}^{\mathbf{w}}$ for most combinations of $i$ and $j$. Explicit dependence on $\mathbf{w}$ is, henceforth, dropped for brevity.

Owing to the GI correction term [S $\mathcal{S}^{\mathrm{GI}}$, Eq. (13)], Eq. (11) cannot be written as a 1-RDM functional. Therefore, the series of steps leading to Eq. (8) cannot be reproduced fully for $\mathcal{S}^{e}$. Instead, one must carry out explicit differentiation with respect to orbitals $\varphi_{i}^{*}$. We first write

$$
\mathcal{C}=\mathcal{S}^{e}[\{\varphi\}]+\mathcal{R}_{S}^{e}[n]-\sum_{i j} f_{i} \varepsilon_{i j}\left[\int \varphi_{i}^{*} \varphi_{j} d \boldsymbol{r}-\delta_{i j}\right]
$$

as our functional to be minimized, where $\mathcal{S}^{e}$ and $\mathcal{R}_{S}^{e}$ are energies, the final term is the constraint that the orbitals be orthonormal, $\left\{f_{i}\right\}$ are average occupation factors, and $\left\{\varepsilon_{i j}=\varepsilon_{j i}^{*}\right\}$ are Lagrange multipliers. Next, we differentiate $\mathcal{C}$ with respect to $\varphi_{i}^{*}$ (which can be treated as independent of $\varphi_{i}$ ) to obtain

$$
\frac{\delta \mathcal{S}^{w}}{\delta \varphi_{i}^{*}(\boldsymbol{r})}+f_{i} \varphi_{i}(\boldsymbol{r}) \frac{\delta \mathcal{R}_{S}^{e}}{\delta n(\boldsymbol{r})}-f_{i} \sum_{j} \varepsilon_{i j} \varphi_{j}(\boldsymbol{r})=0 .
$$

Finally, we rewrite (15) as a series of coupled orbital equations,

$$
\hat{h}_{i} \varphi_{i} \equiv\left\{\hat{t}+v+\hat{v}_{S, i}^{e}+v_{R}^{e}\right\} \varphi_{i}=\sum_{j} \varepsilon_{i j} \varphi_{j},
$$

for all $i, j$ with $f_{i}, f_{j}>0$. Equation (16) must be simultaneously solved to obtain the minimum energy, $\mathcal{E}[n, \mathbf{w}]$. Importantly, because

$$
\hat{v}_{S, i}^{e} \varphi_{i}:=\frac{\delta \mathcal{E}_{\mathrm{Hx}, S}^{e}[\{\varphi\}]}{f_{i} \delta \varphi_{i}^{*}}
$$

varies non-trivially with $i$, a separate one-body problem needs to be solved for each orbital, and Lagrange multipliers $\varepsilon_{i j}$ are required to deal with normality and orthogonality (i.e., $\varepsilon_{i j}\left[\int \varphi_{i}^{*} \varphi_{j} d \boldsymbol{r}-\delta_{i j}\right]$, with $\varepsilon_{i j}=\varepsilon_{j i}^{*}$.

Before proceeding further, it is worth highlighting the similarity between Eq. (16) of the present text and Eqs. (11) and (12) of Filatov. ${ }^{41}$ Both these equations take on the same form as both arise from pair-coefficients that are not products of occupation factors. Here, these equations are derived from a rigorous EGKS theory. Filatov derived them by considering approximate solutions for the spin-restricted ensemble-referenced Kohn-Sham (REKS) approach for strongly correlated systems.

We are now ready to consider Eq. (16) in the titular framework. A solution provably exists and may, thus, formally be found. In this sense, Eq. (16) is a good result. However, it loses many of the appealing features (simple non-local operator, guaranteed orthogonal orbitals) that make GKS useful. Furthermore, it cannot easily be implemented using the existing GKS infrastructure, although more expensive infrastructure, e.g., that used in complete active space selfconsistent field (CASSCF) calculations, may be useful. It is, thus, a bad result, in the sense of offering few obvious practical advantages over the existing approaches [using Eqs. (7) or (8)]. 
In the following, we show that an approximation that is amenable to solution using existing numerical approaches, while still maintaining the gains from both EKS and GKS theories, can be obtained by combining a non-multiplicative potential with an iterative algorithm. Such a theory could be viewed, in comparison to the parent EKS or GKS theory, as ugly. However, we show that it offers formal and practical advantages over existing approaches.

\section{B. More amenable EGKS}

In anticipation of further approximation, we first recast the exact result in a slightly different form. As discussed previously, $S\left[\gamma_{1}^{\mathbf{w}}\right]$ lends itself to standard GKS treatment. We, therefore, "generalize" the KS formalism on this term separately from the difficult $\mathcal{S}^{\mathrm{GI}}$ term [Eq. (13)]. The total energy may be written as

$$
\mathcal{E}[\{\varphi\}]=S\left[\gamma_{1}\right]+\mathcal{S}^{\mathrm{GI}}[\{\varphi\}]+\mathcal{R}_{S}^{e}[n]+V[n] .
$$

This is an exact expression that partitions the straightforward 1-RDM part, $S\left[\gamma_{1}\right]$, in $\mathcal{S}^{e}=S+\mathcal{S}^{\mathrm{GI}}$ from the more difficult ghost interaction term, $\mathcal{S}^{\mathrm{GI}}$.

Our goal is to find the orbitals that minimize $\mathcal{E}$, i.e., solutions of (16). The partitioning is preserved via differentiation, which lets us similarly partition the non-local potential operator,

$$
\hat{v}_{S, i}^{e} \varphi_{i}=\hat{v}_{S} \varphi_{i}+\frac{\delta \mathcal{S}^{\mathrm{GI}}[\{\varphi\}]}{f_{i} \delta \varphi_{i}^{*}}:=\left\{\hat{v}_{S}+\hat{v}_{i}^{\mathrm{GI}}\right\} \varphi_{i} .
$$

Here,

$$
\begin{aligned}
\hat{v}_{S} \varphi_{i} & =\hat{v}_{\mathrm{HF}}\left[\gamma_{1}\right] \varphi_{i}:=v_{\mathrm{H}} \varphi_{i}+\hat{v}_{\mathrm{x}} \varphi_{i} \\
& =\int \frac{n\left(\boldsymbol{r}^{\prime}\right) d \boldsymbol{r}^{\prime}}{\left|\boldsymbol{r}-\boldsymbol{r}^{\prime}\right|} \varphi_{i}(\boldsymbol{r})-\frac{1}{2} \int \frac{\gamma_{1}\left(\boldsymbol{r}, \boldsymbol{r}^{\prime}\right) \varphi_{i}\left(\boldsymbol{r}^{\prime}\right) d \boldsymbol{r}^{\prime}}{\left|\boldsymbol{r}-\boldsymbol{r}^{\prime}\right|}
\end{aligned}
$$

is the usual 1-RDM expression for Hartree-Fock theory that can be used in Eq. (8) without modification by assigning it an ensemble 1RDM.

Our next step is to recognize that $\Delta F_{i j}^{J}=F_{i j}^{J}-f_{i} f_{j}$ and $\Delta F_{i j}^{K}$ $=F_{i j}^{K}+\frac{1}{2} f_{i} f_{j}$ are zero for almost all combinations of $i$ and $j$ in all common types of ensembles, per the Appendix. Therefore, $\frac{\delta \mathcal{S}^{\mathrm{GI}}}{\delta \phi_{i}^{*}}$ is also zero for most values of $i$-including all doubly occupied orbitals with $f_{i}=2$. We define a "doubly occupied space," $\mathbb{D}$, for all orbitals with $f_{i}=2$ and a "frontier space," $\mathbb{F}$, for remaining orbitals with $f_{i}>0$. Furthermore, we can eliminate most of the off-diagonal terms, $\varepsilon_{i \neq j}$ [in (16)], by recognizing that

$$
\varepsilon_{i j}= \begin{cases}\varepsilon_{i} \delta_{i j}, & i, j \in \mathbb{D}, \\ \bar{\omega}_{j}, & i \in \mathbb{D}, j \in \mathbb{F}, \\ \bar{\omega}_{i}^{*}, & i \in \mathbb{F}, j \in \mathbb{D}, \\ \varepsilon_{i j}, & i, j \in \mathbb{F},\end{cases}
$$

which follows from the fact that $\hat{v}_{S}$ is Hermitian, $\varepsilon_{i j}=\varepsilon_{j i}^{*}$, and $\gamma_{1}$ and $\mathcal{S}^{1-\mathrm{RDM}}\left[\gamma_{1}\right]$ are invariant to unitary transformations among $i \in \mathbb{D}$.

It finally follows from Eqs. (13), (19), and (21) that

$$
\begin{array}{cc}
\hat{h}_{1-\mathrm{RDM}} \varphi_{i}=\varepsilon_{i} \varphi_{i}+\sum_{j \in \mathbb{F}} \bar{\omega}_{j} \varphi_{j}, & i \in \mathbb{D}, \\
\left\{\hat{h}_{1-\mathrm{RDM}}+\hat{v}_{i}^{\mathrm{GI}}\right\} \varphi_{i}=\sum_{j \in \mathbb{F}} \varepsilon_{i j} \varphi_{j}+\bar{\omega}_{i}^{*} \sum_{j \in \mathbb{D}} \varphi_{j}, & i \in \mathbb{F},
\end{array}
$$

where $\hat{h}_{1-\mathrm{RDM}}:=\hat{t}+v+\hat{v}_{S}+v_{R}$ and

$$
\begin{aligned}
\hat{v}_{i}^{\mathrm{GI}}= & \sum_{j} \frac{\Delta F_{i j}^{J}}{f_{i}} \int \frac{n_{j}\left(\boldsymbol{r}^{\prime}\right) d \boldsymbol{r}^{\prime}}{\left|\boldsymbol{r}-\boldsymbol{r}^{\prime}\right|} \varphi_{i}(\boldsymbol{r}) \\
& +\sum_{j} \frac{\Delta F_{i j}^{K}}{f_{i}} \int \frac{\varphi_{j}^{*}\left(\boldsymbol{r}^{\prime}\right) \varphi_{i}\left(\boldsymbol{r}^{\prime}\right) d \boldsymbol{r}^{\prime}}{\left|\boldsymbol{r}-\boldsymbol{r}^{\prime}\right|} \varphi_{j}(\boldsymbol{r}) .
\end{aligned}
$$

Simultaneously solving Eq. (22) (for doubly occupied orbitals) and (23) (separately for each frontier orbital) yields the orbitals that minimize Eq. (18).

\section{Practical approximations}

Solving Eqs. (22) and (23) is difficult in general and cannot be trivially performed using standard iterative approaches to GKS theory implemented in quantum chemistry codes. We therefore propose two approximations that can be solved by using existing machinery directly, supplemented by simple linear algebra routines that are present in any quantum chemistry code.

A first, crude approximation is to assume that $\hat{v}_{i}^{\mathrm{GI}}$ is of little consequence to the obtained EGKS orbitals and so can be set to zero. We therefore introduce the "1-RDM" approximation, in which the orbitals $\{\varphi\}_{1-\mathrm{RDM}}$ are found using

$$
\hat{h}_{1-\mathrm{RDM}} \varphi_{i}^{1-\mathrm{RDM}}=\varepsilon_{i}^{1-\mathrm{RDM}} \varphi_{i}^{1-\mathrm{RDM}},
$$

but energies are found by using $\{\varphi\}_{1-\mathrm{RDM}}$ in Eq. (18). Here, the offdiagonal terms disappear naturally because $\hat{h}_{1-\mathrm{RDM}}$ is Hermitian. The ensemble energy from this approximation provides an upper bound to the true EGKS energy.

On the positive side, the 1-RDM approximation is simple and convenient. On the negative side, it is uncontrolled, misses key ensemble physics, and even leads to asymptotically incorrect potentials-see the $\mathrm{Li}^{+q}$ example in Sec. IV. Nonetheless, it is a decent approximation in some cases, such as for the cation of HCN (shown in Fig. 1).

The second, "diagonal" approximation represents the titular ugly approach. It involves setting all off-diagonal terms in Eq. (21) to zero, not just the ones for $i, j \in \mathbb{D}$. That is, it involves solving

$$
\begin{aligned}
& \hat{h}_{1-\operatorname{RDM}} \varphi_{i}^{\mathrm{diag}}=\varepsilon_{i}^{\mathrm{diag}} \varphi_{i}^{\mathrm{diag}}, \quad i \in \mathbb{D}, \\
& \left\{\hat{h}_{1-\mathrm{RDM}}+\hat{v}_{i}^{\mathrm{GI}}\right\} \varphi_{i}^{\mathrm{diag}}=\varepsilon_{i}^{\mathrm{diag}} \varphi_{i}^{\mathrm{diag}}, \quad i \in \mathbb{F},
\end{aligned}
$$

and algorithmically imposing orthogonality. The resulting solution has better properties than the 1-RDM solution and, in almost all tested cases, gives lower energies. The orthogonalization and need to solve multiple equations, nonetheless, make it rather ugly compared to standard GKS theory of Eq. (8).

The difference between the 1-RDM and diagonal approximations is best illustrated using a finite $M$-element real basis set, so that $\varphi_{i} \rightarrow \boldsymbol{C}_{i}$ are the orbitals and $\gamma_{1}=\sum_{i} f_{i} \varphi_{i}^{*}(\boldsymbol{r}) \varphi_{i}\left(\boldsymbol{r}^{\prime}\right) \rightarrow \boldsymbol{D}=\sum_{i} f_{i} \boldsymbol{C}_{i} \boldsymbol{C}_{i}^{T}$ is the 1-RDM. Then, $\hat{h}_{1-\mathrm{RDM}}\left[\gamma_{1}\right] \rightarrow \boldsymbol{F}_{1-\mathrm{RDM}}[\boldsymbol{D}]$ and $\hat{v}_{i}^{\mathrm{GI}}\left[\{\varphi\}_{i}\right] \rightarrow$ $\boldsymbol{V}\left[\left\{\boldsymbol{C}_{i}\right\}\right]_{i}^{\mathrm{GI}}$ are symmetric matrix representations of effective Hamiltonian terms, expressed in the basis. We also introduce $S$ as the $M \times M$ overlap matrix of our basis, to deal with non-orthogonal choices.

For both approximations we first solve

$$
\boldsymbol{F}_{1-\mathrm{RDM}} \boldsymbol{C}_{i}^{1-\mathrm{RDM}}=\varepsilon_{i} S \boldsymbol{C}_{i}^{1-\mathrm{RDM}}
$$



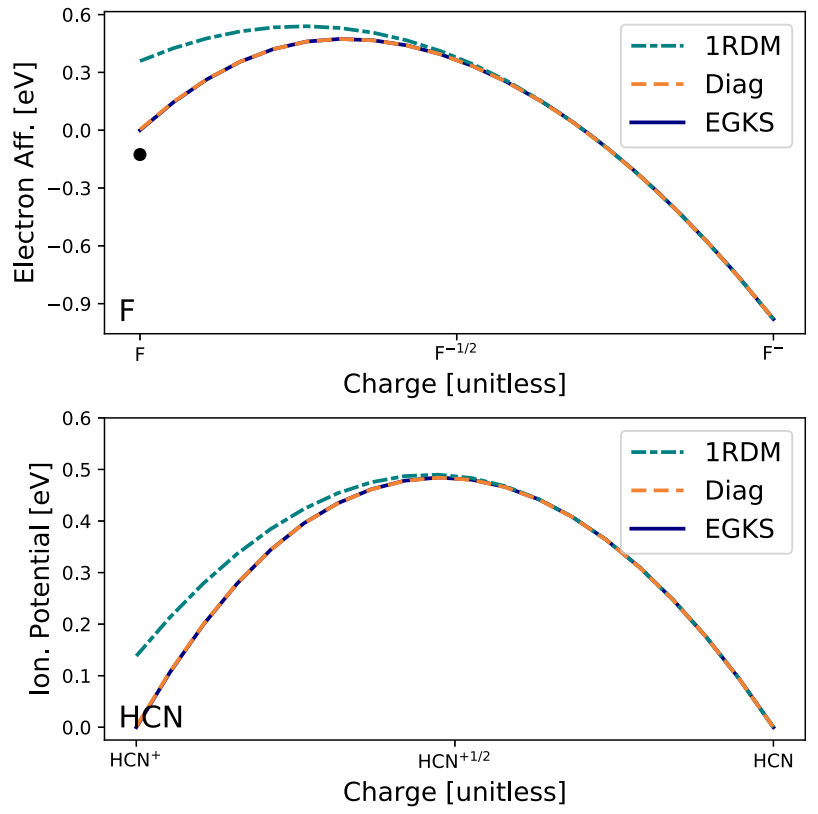

FIG. 1. Fractional anion energy curve of $F(t o p)$ shown relative to the neutral atom energy of EGKS theory and fractional cation curve of $\mathrm{HCN}$ (bottom), shown relative to a straight line fit between the neutral and cation EGKS results. Here and in subsequent figures, we show 1-RDM in teal dash-dots, diag in orange dashes, and exact EGKS results in navy solid lines. Dots, where shown, indicate UHF values.

for all orbitals such that $C_{i}^{1-\mathrm{RDM}}$ are guaranteed to be orthogonal. The 1-RDM approximation then involves updating $\boldsymbol{F}_{1-\mathrm{RDM}}$ using $\boldsymbol{D}^{1-\mathrm{RDM}}$ from these orbitals and solving to self-consistency. Similarly, in the diagonal approximation, the solutions, $C_{i \in \mathbb{D}}^{\text {diag }}:=C_{i}^{1-\mathrm{RDM}}$, are used for our doubly occupied orbitals. The difference comes because the solutions, $C_{i \notin \mathbb{D}}^{1-\mathrm{RDM}}$, are used to expand the frontier orbitals, $\boldsymbol{C}_{i \in \mathbb{F}}^{\mathrm{diag}}$, which are then solved separately.

To solve for $C_{i \in \mathbb{F}}^{\mathrm{diag}}$, we must first impose an order on $i \in \mathbb{F}$, e.g., via $\varepsilon_{i}^{\text {diag }}$ from a previous step or the ground state. For the first $i \in \mathbb{F}$, we define $\boldsymbol{P}^{i}=\left[\boldsymbol{C}_{i}^{1-\mathrm{RDM}}, \boldsymbol{C}_{i+1}^{1-\mathrm{RDM}}, \ldots\right]$ (i.e., a matrix formed out of the frontier and virtual orbitals expressed as column vectors) that projects solutions onto the unoccupied space of Eq. (28). We then diagonalize

$$
\boldsymbol{P}^{i, T}\left\{\boldsymbol{F}_{1-\mathrm{RDM}}+\boldsymbol{V}_{i}^{\mathrm{GI}}\right\} \boldsymbol{P}^{i} \boldsymbol{x}_{k}=\varepsilon_{k}^{i} \boldsymbol{x}_{k},
$$

to obtain $\boldsymbol{C}_{k}^{i}:=\boldsymbol{P}^{i} \boldsymbol{x}_{k}$, ordered for $k \geq 1$. Then, $\boldsymbol{C}_{i}^{\mathrm{diag}}:=\boldsymbol{C}_{k=1}^{i}$ is the next orbital for the diagonal approximation. We then set $\boldsymbol{P}^{i+1}$ $=\left[\boldsymbol{C}_{2}^{i}, \boldsymbol{C}_{3}^{i}, \ldots\right]$ and solve Eq. (29) again for $i+1$, until we have obtained all $i \in \mathbb{F}$. Once we have the full set of orbitals, we can use them to update $\boldsymbol{F}_{1-\mathrm{RDM}}\left[\boldsymbol{D}^{\mathrm{diag}}\right]$ and $\boldsymbol{V}_{i}^{\mathrm{GI}}\left[\left\{\boldsymbol{C}_{i}^{\mathrm{diag}}\right\}\right]$ and then use the new matrices to repeat the process starting from Eq. (28).

The approach, thus, gives us a set of orbitals, $C_{i}^{\text {diag }}$, that are guaranteed to be orthogonal to one another. Furthermore, the use of $\hat{v}_{i}^{\mathrm{GI}}$ in the effective Hamiltonian for $\boldsymbol{C}_{i}^{\mathrm{diag}}$ means that they, prima facie, have the correct asymptotics, ${ }^{67}$ unlike the 1-RDM solutions. They may, thus, be expected to be better approximations to the real solutions. Results (see Sec. IV) are almost always better than the 1RDM, sometimes substantially so. Nevertheless, they can only form an upper bound to the true energy since $\varepsilon_{i \neq j}=0$ is imposed via the diagonal approximation, rather than found from a true variational solution.

Of final note, it is obvious that the frontier orbitals, $C_{i \in \mathbb{F}}^{\text {diag }} \neq$ $C_{i}^{1-\mathrm{RDM}}$, differ from the 1-RDM approximation because they obey different effective Hamiltonians. However, it is also true that the doubly occupied orbitals, $\boldsymbol{C}_{i \in \mathbb{D}}^{\text {diag }} \neq \boldsymbol{C}_{i}^{1-\mathrm{RDM}}$, are different despite both coming from Eq. (28). The difference comes from different 1-RDMs, $\boldsymbol{D}^{\text {diag }} \neq \boldsymbol{D}^{1-\mathrm{RDM}}$, being used to form $\boldsymbol{F}_{1 \text {-RDM }}$.

\section{Simplified EGKS theory}

We next turn briefly to a less rigorous approximation recently introduced by one of the authors-the "simplified EGKS" (SEGKS) scheme $^{60}$ - that is designed to efficiently approximate the EGKS solution of excited state ensembles. This approximation is based on an ansatz, $\boldsymbol{F} \approx(1-\mu) \boldsymbol{F}_{\mathrm{gs}}+\mu \boldsymbol{F}_{h \rightarrow l}$, for the Fock matrix that interpolates between the ground state Fock matrix, $\boldsymbol{F}_{\mathrm{gs}}$, and a different Fock matrix, $\boldsymbol{F}_{h \rightarrow l}$, formed by double promotion of the HOMO to the LUMO.

The physical intuition behind this ansatz is the idea that $\boldsymbol{F}_{\mathrm{gs}}$ screens the HOMO more than the LUMO, whereas $\boldsymbol{F}_{h \rightarrow l}$ does the reverse, which gives the model sufficient flexibility to optimize the orbitals. The precise amount of linear mixing is found by seeking $\mu^{s}$, for which the resulting orbitals, $C_{i}^{s}$, minimize the total energy, $\mathcal{E}^{s}$, including the GI term. SEGKS provides an upper bound to the true EGKS energy.

\section{E. A very slow route to exact solutions}

Before concluding this section, we return to exact theory, where our goal is to find the orbitals, $\boldsymbol{C}_{i}^{o}$, that minimize Eq. (18), to provide the best possible energy, $\mathcal{E}^{o}:=\mathcal{E}\left[\left\{\boldsymbol{C}_{i}^{o}\right\}\right] \leq \mathcal{E}\left[\left\{\boldsymbol{C}_{i}\right\}\right]$, within the given finite basis. In all tested cases, we found that $\boldsymbol{C}_{i}^{\text {diag }}$ forms a good starting point for finding $C_{i}^{o}$, in the sense that we may write $\boldsymbol{C}_{i}^{o}=\sum_{j} U_{i j} \boldsymbol{C}_{j}^{\mathrm{diag}}$, where the unitary matrix $\boldsymbol{U}$ (in our finite basis) is nearly an identity. Consequently, we may write $\boldsymbol{U} \approx \prod_{i>j} \boldsymbol{u}_{i j}\left(\theta_{i j}\right)$, where $\boldsymbol{u}_{i j}\left(\theta_{i j}\right)$ are matrices that leave all orbitals unchanged except $\boldsymbol{C}_{i} \rightarrow \cos \left(\theta_{i j}\right) \boldsymbol{C}_{i}-\sin \left(\theta_{i j}\right) \boldsymbol{C}_{j}$ and $\boldsymbol{C}_{j} \rightarrow \sin \left(\theta_{i j}\right) \boldsymbol{C}_{i}+\cos \left(\theta_{i j}\right) \boldsymbol{C}_{j}$.

We are, thus, able to inefficiently find $\mathcal{E}^{o}$ by individually minimizing the energy with respect to rotations, $\theta_{i j}$, for each combination of $j>i$ (including doubly occupied, frontier, and virtual orbitals but excluding double-double and virtual-virtual rotations as these leave the energy unchanged). This brute force approach leads to $O\left(N^{2}\right)$ calculations of $O\left(N^{3}\right)$ energies, for a very poor scaling of $O\left(N^{5}\right)$ that is unsuitable for large systems. In practice, we repeat the process several times until energies can no longer be reduced. We are, thus, able to find exact solutions, to within a few percent of an $\mathrm{eV}$, for the small systems reported in Sec. IV.

\section{RESULTS}

Having established both exact theory and useful approximate forms, we turn to examples. Throughout, we perform EGKS calculations corresponding to the ensemble exact exchange theory approximation $^{56}$ in EKS theory, i.e., we set $\mathcal{R}^{e}=0, \mathcal{S}^{1-\mathrm{RDM}} \equiv \mathcal{S}^{\mathrm{HF}}$, and 
$\mathcal{S}^{\mathrm{GI}}=\mathcal{T}_{s}+\mathcal{E}_{\mathrm{Hx}}-\mathcal{S}^{\mathrm{HF}}$. As mentioned above, we use a spinunpolarized formalism throughout so that potentials and orbitals are independent of spin, i.e., $\varphi_{i \uparrow}=\varphi_{i \downarrow}$ and $\boldsymbol{C}_{i \uparrow}=\boldsymbol{C}_{i \downarrow}$.

For the purpose of the present theoretical study, exact exchange represents an effective choice of density functional approximation as it lets us clearly delineate errors caused by the orbital approximations (1-RDM or diag) from those caused by the functional approximation. We are, thus, able to gain insights into the quality of approximations to the effective Hamiltonians (and orbitals), separate from other considerations. These calculations are denoted as "exchangeonly" throughout, and the underlying orbital approximation (EGKS, diag, 1-RDM, or unrestricted Hartree-Fock) are mentioned separately. For a similar purpose, we also break spatial symmetries, by arbitrarily occupying selected degenerate orbitals in all cases, to avoid additional ensemble effects required to deal with them. ${ }^{28,38,68}$

We begin with an analytic example: the fractional ion $\mathrm{Li}^{+q}$, with $0<q<1$. This system is represented as an ensemble $\hat{\Gamma} \equiv q\left|1 s^{2}\right\rangle\left\langle 1 s^{2}\right|$ $+(1-q) / 2\left[\left|1 s^{2} 2 s^{\uparrow}\right\rangle\left\langle 1 s^{2} 2 s^{\uparrow}|+| 1 s^{2} 2 s^{\downarrow}\right\rangle\left\langle 1 s^{2} 2 s^{\downarrow}\right|\right]$. It is straightforward to show (see Appendix) that the density is $n=2\left|\phi_{1 s}\right|^{2}+(1-q)\left|\phi_{2 s}\right|^{2}$, i.e., $f_{1 s}=2$ and $f_{h} \equiv f_{2 s}=1-q$. Furthermore, the $2 s$ orbital never interacts with itself, which gives $F_{2 s 2 s}^{J}=F_{2 s 2 s}^{K}=0$ as the only paircoefficient that contributes to

$$
\mathcal{S}^{\mathrm{GI}}[\{\varphi\}]:=\mathcal{S}^{\mathrm{GI}}\left[\phi_{2 s}\right]=-\frac{f_{2 s}^{2}}{2} E_{\mathrm{H}}\left[n_{2 s}\right] .
$$

We are interested in the asymptotic behavior of the $2 s$ orbital. By recognizing that for large $r \int \frac{\varphi_{i}^{*}\left(r^{\prime}\right) \varphi_{j}\left(r^{\prime}\right) d r^{\prime}}{\left|r-r^{\prime}\right|} \rightarrow \frac{\delta_{i j}}{r}$, we see [Eq. (20)] that $v_{\mathrm{H}}^{1-\mathrm{RDM}} \varphi_{2 s}=\int \frac{n\left(\boldsymbol{r}^{\prime}\right)}{\left|\boldsymbol{r}-\boldsymbol{r}^{\prime}\right|} d \boldsymbol{r}^{\prime} \varphi_{2 s}(\boldsymbol{r}) \rightarrow \frac{f_{1 s}+f_{2 s}}{r} \varphi_{2 s}$ and $\hat{v}_{\mathrm{x}}^{1-\mathrm{RDM}} \varphi_{2 s}=-\int \frac{\gamma_{1}\left(\boldsymbol{r}, \boldsymbol{r}^{\prime}\right)}{2\left|\boldsymbol{r}-\boldsymbol{r}^{\prime}\right|} \varphi_{2 s}\left(\boldsymbol{r}^{\prime}\right) d \boldsymbol{r}^{\prime} \rightarrow-\frac{f_{2 s}}{2 r} \varphi_{2 s}$ so that the 1-RDM approximation [Eq. (25)] yields

$$
\lim _{r \rightarrow \infty} \hat{v}_{S} \varphi_{2 s} \rightarrow \frac{f_{1 s}+\frac{f_{2 s}}{2}}{r} \varphi_{2 s}=\frac{2+\frac{1-q}{2}}{r} \varphi_{2 s} .
$$

Including the GI term [Eq. (30) in (24)], whether in the exact theory or via the diagonal approximation [Eqs. (26) and (27)], gives an additional $\hat{v}_{2 s}^{\mathrm{GI}} \rightarrow-\frac{f_{2 s}^{2}}{2 f_{2 s} r}=\frac{-f_{2 s}}{2 r}$, to yield

$$
\lim _{r \rightarrow \infty}\left[\hat{v}_{S}+\hat{v}_{2 s}^{\mathrm{GI}}\right] \varphi_{2 s} \rightarrow \frac{f_{2 s}}{r} \varphi_{2 s}=\frac{2}{r} \varphi_{2 s} .
$$

Note that we have assumed $\lim _{r \rightarrow \infty} \varphi_{1 s}(\boldsymbol{r}) / \varphi_{2 s}(\boldsymbol{r})=0$.

The outermost $2 s$ electron, thus, "feels" the charge of the two $1 s$ electrons only (i.e., no self-interaction) when the GI term is included, a result that is physical and consistent with previous findings of ensemble theories yielding the correct asymptotic behavior in ensembles. ${ }^{68}$ By contrast, the 1-RDM approximation leads to an unphysical self-interaction in the outermost electron, with an effective charge $\frac{q-1}{2}$, and, thus, underbinds the $2 s$ orbital. The total effective asymptotic potential (nuclear plus electronic) felt by the outermost electron is, thus, $-\frac{1}{r}$ for exact GKS and the diagonal approximation, but $\frac{-1-q}{2 r}$ for the 1-RDM approximation.

The abovementioned result is not unique to the case $f_{h} \leq 1$. In general, exact EGKS theory and the diag approximation for fractional anions and cations will have asymptotic behavior in line with known OEP results. ${ }^{68}$ This result follows from the fact that OEPs, exact GKS theory, and the diag approximation all reduce to the same
Slater potential in the limit $r \rightarrow \infty$ and, thus, have the same asymptotic behavior. However, this behavior is not necessarily that of a $\frac{-1}{r}$ decay. ${ }^{68}$ The 1-RDM approximation always decays asymptotically as $\frac{-Z+N-f_{h} / 2}{r}$ where $Z$ is the nuclear charge and $N$ is the total number of electrons.

We now turn to numerical examples, where we compare our two approximations (1-RDM and diag) against benchmark exchange-only solutions found by minimizing against unitary transformations of the orbitals, as described in Sec. III E. All calculations were performed using Gaussian type orbitals in the def2-TZVP basis set $^{69}$ and were implemented in a customized Python 3 code using Psi4/numpy. ${ }^{70,71}$ We remind the reader that, in all examples, we have allowed spatial symmetries to break.

Table I reports errors for a selection of simple systems with degenerate ground states (triplet states, ts and doublet states, ds), shown relative to exact EGKS theory. In the case of CO, we show the excited triplet state. These systems have the advantage that not only can we compare exchange-only EGKS approximations against exact results but also compare exact and approximate EGKS against broken-symmetry unconstrained Hartree-Fock (UHF) theory. UHF is guaranteed to have an energy that is less than or equal to EGKS by virtue of having additional degrees of freedom. It is, therefore, included as a reference lower bound value for exchange-only theories.

We first notice that the diagonal approximation offers significant improvements over the 1-RDM approximation in all tested cases-an unsurprising success that is replicated in almost all tests reported here. Perhaps more surprisingly, we see that even exact EGKS theory can yield substantially larger energies than UHF, of up to $8.9 \mathrm{kcal} / \mathrm{mol}(0.38 \mathrm{eV})$ for the $\mathrm{CO}$ excited triplet state. Whether this should be considered an "error" of EGKS (which has the correct ground state degeneracy but higher energy) or UHF (which lowers energies via an unphysical breaking of fundamental symmetries) is a matter of taste that dates back to, at least, 1963 as Löwdin's classic "symmetry dilemma."

A more comprehensive example is provided by the full fractional ionization curve for the anion of the fluorine atom, which represents the zero temperature limit of a grand canonical ensemble. At net charge $q$, the ensemble describing this process is

$$
\hat{\Gamma}_{q}=\frac{1+q}{2}\left[\left|\mathrm{~F}_{\uparrow}\right\rangle\left\langle\mathrm{F}_{\uparrow}|+| \mathrm{F}_{\downarrow}\right\rangle\left\langle\mathrm{F}_{\downarrow}\right|\right]-q\left|\mathrm{~F}^{-}\right\rangle\left\langle\mathrm{F}^{-}\right|,
$$

which mixes equal amounts of the $\uparrow$ and $\downarrow$ dominant degenerate $\mathrm{F}$ doublet ground states with the singlet $\mathrm{F}^{-}$ground state, to achieve the correct net charge. Results are shown in the top panel of Fig. 1.

TABLE I. Errors (in $\mathrm{kcal} / \mathrm{mol}=0.043 \mathrm{eV}$ ) for various EGKS approximations, with respect to exact EGKS energies, within exchange-only theory. Also shown are energies from UHF theory, for triplet state (ts) and doublet states (ds). Singlet states are identical in all theories.

\begin{tabular}{lrrrrrr}
\hline \hline & $\mathrm{C}(\mathrm{ts})$ & $\mathrm{O}(\mathrm{ts})$ & $\mathrm{B}(\mathrm{ds})$ & $\mathrm{F}(\mathrm{ds})$ & $\mathrm{CO}(\mathrm{ts})$ & Mean \\
\hline 1-RDM & 11.6 & 15.6 & 5.2 & 8.3 & 14.1 & 11.0 \\
Diag & 4.0 & 7.7 & 0.0 & 0.1 & 6.3 & 3.6 \\
Exact & 0.0 & 0.0 & 0.0 & 0.0 & 0.0 & 0.0 \\
UHF & -3.1 & -3.9 & -2.7 & -2.9 & -8.9 & -4.3 \\
\hline \hline
\end{tabular}


In a correlated theory, this curve should be a straight line. ${ }^{22}$ Exchange-only theory introduces a substantial curvature as a function of charge. ${ }^{28,73,74}$ It is, nonetheless, clear that the diagonal approximation is nearly exact for all charges, whereas the 1-RDM approximation is only accurate when more than half an electron is added to the system; note that $\mathrm{F}^{-}$is the closed shell pure state case for which standard 1-RDM theories apply so that exact, 1-RDM, and diag EGKS all become exactly equivalent to standard Hartree-Fock theory.

The bottom panel of Fig. 1 is similar to the top, but for the cation of $\mathrm{HCN}$,

$\hat{\Gamma}_{q}=\frac{q}{2}\left[\left|\mathrm{HCN}_{\uparrow}^{+}\right\rangle\left\langle\mathrm{HCN}_{\uparrow}^{+}|+| \mathrm{HCN}_{\downarrow}^{+}\right\rangle\left\langle\mathrm{HCN}_{\downarrow}^{+}\right|\right]+(1-q)|\mathrm{HCN}\rangle\langle\mathrm{HCN}|$.

Rather than showing full energies, it reports errors relative to a straight line fit between exchange-only EGKS results for the cation and neutral molecule, i.e., it shows the curvature, so that difference between approximations are visible at the scale of the plot. Again diag is nearly exact, whereas 1-RDM has issues for positive charges of more than half an electron-here, the neutral system is the closed shell case.

EDFT can also be used to predict excitations energies. This is performed by forming an ensemble in which the excited states have weights less than or equal to the weights for the states that are lower in energy. We, therefore, next consider a relevant ensemble for predicting singlet-triplet excitations,

$$
\hat{\Gamma}_{w}=(1-w)\left|S_{0}\right\rangle\left\langle S_{0}\left|+\frac{w}{3} \sum_{M_{z}}\right| T_{0, M_{z}}\right\rangle\left\langle T_{0, M_{z}}\right|,
$$

which mixes different fractions of the singlet ground state (gs, $S_{0}$ ) with an equal mixture of the three lowest energy triplet states (ts, $T_{0, M_{z}}, M_{z} \in\{-1,0,1\}$ ). Here, $w=0$ indicates a pure ground (singlet) state while $w=1$ represents the state average of the degenerate triplets.

It is important to note here that the original Gross-OliveiraKohn theorem ${ }^{35}$ that underlies excited state ensembles applies to systems with non-increasing weights, $w_{\kappa}$, as a function of energy, $E_{\kappa}$, and, thus, forbids the ensemble with $w>\frac{3}{4}$ because $w_{\mathrm{ts}}=\frac{w}{3}>$ $1-w=w_{\mathrm{gs}}$ even though $E_{\mathrm{ts}}>E_{\mathrm{gs}}$. However, seamless inclusion of $w>\frac{3}{4}$ is allowed under generalized ensemble theorems ${ }^{38}$ because the ensemble includes only the lowest energy state for each included spin symmetry.

Results are shown in Fig. 2 for Be, C, and O. In all cases, the diag approximation is substantially better than the 1-RDM, except for the pure singlet ground state where both approximations are exact. The diag results are nearly exact for mixings up to $60 \%$ of triplet, but then become poorer. We also include results from the SEGKS scheme. ${ }^{60}$ In all cases, SEGKS outperforms 1-RDM slightly, especially for midrange weights, but is worse than the diag approximation. Note that previous EDFT calculations of Be yielded a singlet-triplet excitation energy of between $2.3 \mathrm{eV}$ and $3.5 \mathrm{eV},{ }^{44,45}$ using a variety of exchangecorrelation models. Although these are larger than the $1.5 \mathrm{eV}$ found here, at least, some of the difference can be explained by the fact that correlations are completely neglected in our calculations.

The same scheme described above for triplet states can also be used to describe excitations between states of the same fundamental symmetry, which are not accessible to standard DFT. Ensembles that
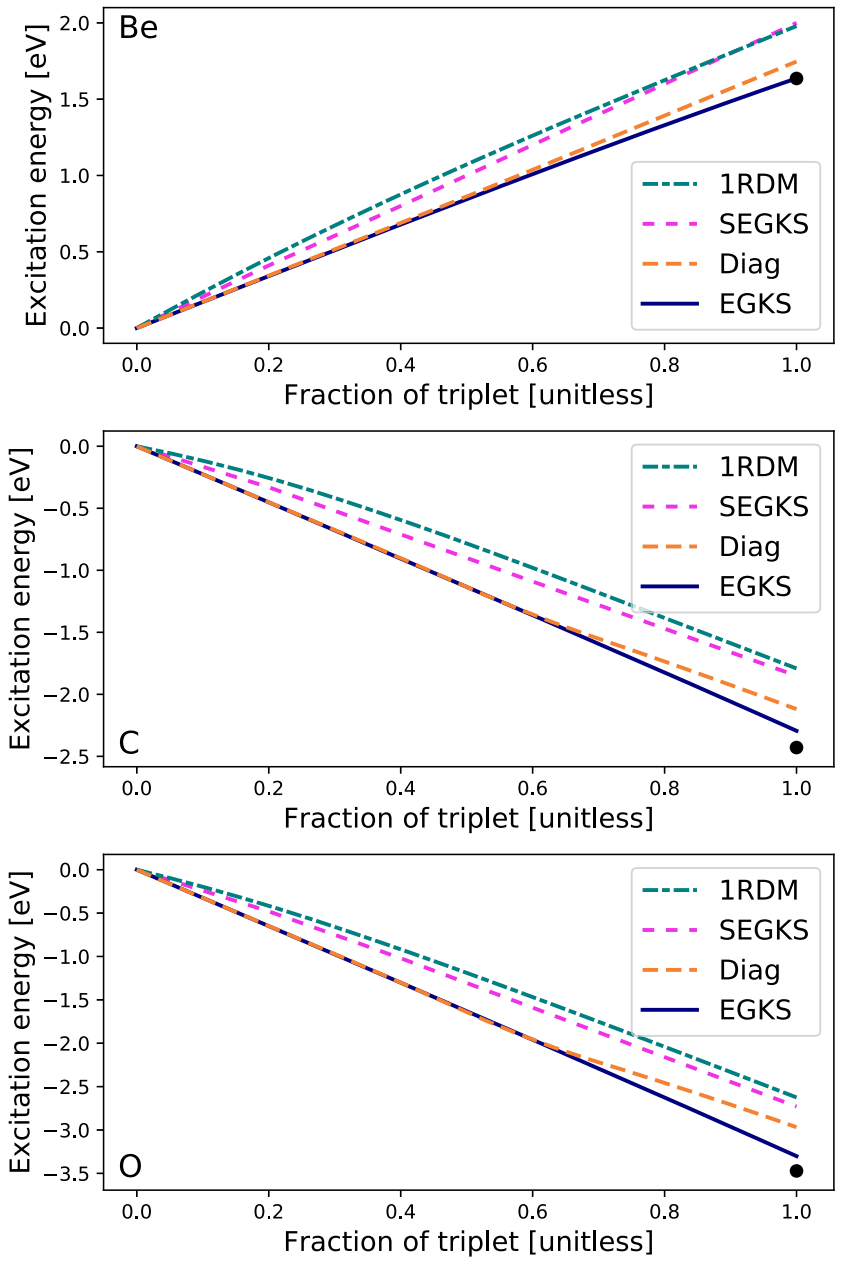

FIG. 2. Energies (in eV) of mixtures of singlet and triplet states for Be (top), C (middle), and $\mathrm{O}$ (bottom).

provide access to the first and second excited states (for single and double excitations from $h \rightarrow l$ ) are

$$
\begin{gathered}
\hat{\Gamma}_{w \leq \frac{1}{2}}=(1-w)\left|S_{0}\right\rangle\left\langle S_{0}|+w| S_{1}\right\rangle\left\langle S_{1}\right|, \\
\hat{\Gamma}_{\frac{1}{2}<w \leq 1}=\frac{2-w}{3}\left[\left|S_{0}\right\rangle\left\langle S_{0}|+| S_{1}\right\rangle\left\langle S_{1}\right|\right]+\frac{2 w-1}{3}\left|S_{2}\right\rangle\left\langle S_{2}\right|,
\end{gathered}
$$

where again we use generalized ensemble theorems, ${ }^{38}$ in this case to eliminate the triplets from the abovementioned ensembles. Here, setting $w \leq \frac{1}{2}$ gives mixtures of $S_{0}$ and $S_{1}$ only, whereas $\frac{1}{2}<w \leq 1$ mixes $S_{2}$ with an equal mixture of $S_{0}$ and $S_{1}$. It, thus, provides a means of obtaining both single and double excitation energies. Ensembles of the form proposed for $w \leq \frac{1}{2}$ were recently used by one of the authors to obtain accurate singlet-singlet excitation energies of small molecules, using SEGKS together with standard density functional approximations.

Figure 3 shows results for excitations of $\mathrm{HCN}$ (top) and $\mathrm{C}_{2}$ (bottom), the latter of which has very small gaps and a degenerate 

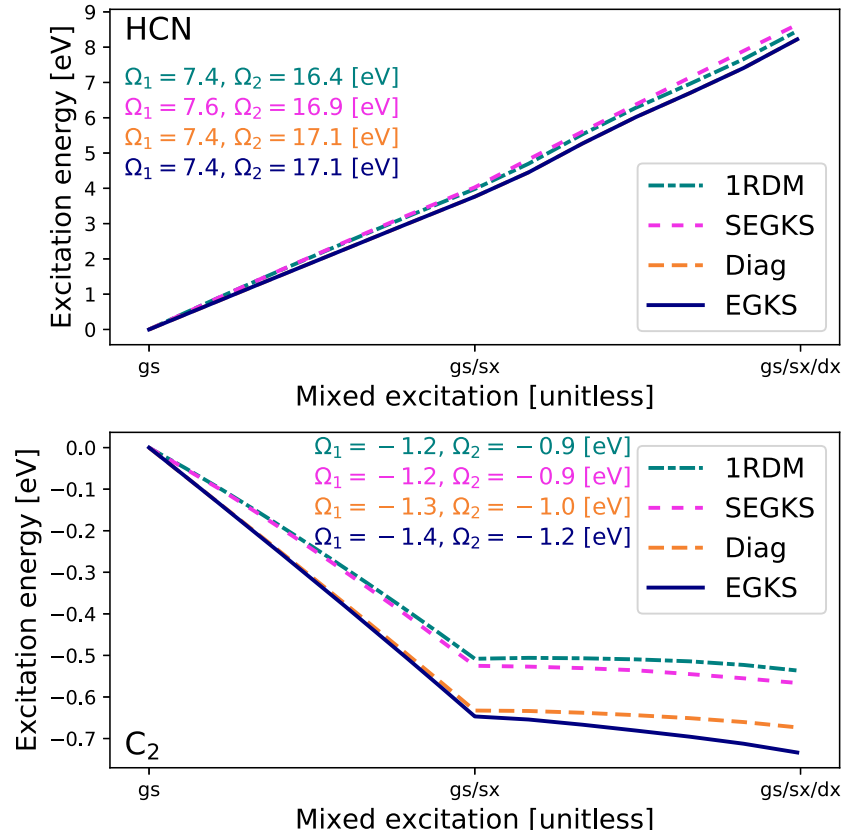

FIG. 3. Energies (in eV) for mixtures of singlet excitations for HCN (top) and $\mathrm{C}_{2}$ (bottom). The $\Omega$ values show extrapolated excitation energies in the different approaches, using the same colors and order as the legend.

HOMO, which we treat here as non-degenerate by breaking spatial symmetry (equivalent to applying an infinitesimal electric field in a direction perpendicular to the bond). In both cases, we see that diag outperforms 1-RDM for all values of $w$, with the diagonal approximation being nearly exact for $\mathrm{HCN}$ but a little poorer for double excitations of $\mathrm{C}_{2}$. SEGKS once again slightly out-performs 1$\mathrm{RDM}$, but is worse than diag. For HCN, the curvature is rather small compared to the overall energy, whereas for $\mathrm{C}_{2}$, it is significant.

Note that the categorization of $S_{0}, S_{1}$, and $S_{2}$ here is based on pure state orbital ordering, not energies. For $\mathrm{C}_{2}$ in the exchange-only (and non-degenerate) approximation, the order is reversed, as seen by the negative energies in the figure. Further note that the nature of the ensemble used for these calculations changes discontinuously at $w=\frac{1}{2}$, at which point the second excited state is introduced. This leads to derivative discontinuities for properties that depend on $w$; in our plots, this is revealed via discontinuities in the exact and approximate energies at $w=\frac{1}{2}$.

The abovementioned ensembles can be used further to estimate excitation gaps. This is performed via extrapolation, by using a quadratic fit to the energy to approximate the energy of a desired excited pure state. ${ }^{38,60}$ The energy, $E_{S_{0}}$, of the ground state $S_{0}$ is found by setting $w=0$. We extrapolate results for $w \leq \frac{1}{2}$ to $w=1$ to obtain the energy, $E_{S_{1}}$, of the $S_{1}$ state and extrapolate $\frac{1}{2} \leq w<1$ to $w=2$ to obtain the energy, $E_{S_{2}}$, of $S_{2}$. The excitation energies, $\Omega_{1}=E_{S_{1}}-E_{S_{0}}$ and $\Omega_{2}=E_{S_{2}}-E_{S_{0}}$, are included in the curves, for each method. Keep in mind that these values are rather poor, due to our choice to use an exchange-only approximation that completely neglects any correlations. For this reason, we do not include exact values but rather compare against the exchange-only benchmark value provided by EGKS theory.

Because the energy functional employed in all calculations reported here is the same, differences between approximations and with exact theories come from the orbitals. Thus, to further understand differences between the approximations, we show, in Fig. 4, the highest occupied and lowest unoccupied molecular orbital densities (HOMO and LUMO, $h$ and $l$ ) of $\mathrm{C}_{2}$ under the two approximations and in exact EGKS. We set $w=1$ to obtain an equal mixing of $S_{0}, S_{1}$, and $S_{2}$. The top panel shows contours $\left(10^{-4}\right.$ and $\left.10^{-2}\right)$ of angular integrals, $\rho(z, r)=\frac{1}{2 \pi} \int_{0}^{2 \pi} \rho(\boldsymbol{r}) d \theta$, while the bottom panel shows $\rho(z)=2 \pi \int_{0}^{\infty} r \rho(z, r) d r$.

It is clear that the diagonal approximation yields better densities than the 1-RDM, as expected from the better energies. What is interesting is that neither approximation manages to shift sufficient charge from outside the molecule to the bond, in the HOMO. This can be seen best by comparing the approximations against exact EGKS for $z \approx 0$, in the bottom panel. The diag does a better, albeit imperfect, job than the 1-RDM. The failure of the diag approximation suggests that GI effects can be quite complicated and non-local, since the only assumption in the diag approximation is that different orbitals do not couple together via $\bar{\omega}_{j}$ and $\epsilon_{i \neq j}$.

Finally, we turn to a rare example where the diag approximation is higher in energy than the 1-RDM: the first singlet-excitation of Be. Figure 5 shows the errors of the diag and 1-RDM approximations for the same excitation process as in Fig. 3. Here, the broken symmetry leads to a unique $2 s 2 p_{z}$ singlet state, which we mix with a $2 p_{z}^{2}$ doubly excited state by taking advantage of generalized ensemble theorems again. ${ }^{3}$

When the amount of $S_{0}$ and $S_{1}$ is approximately equal $\left(w \rightarrow \frac{1}{2}\right)$, the 1-RDM approximation is actually better than the diag, although it is worth noting that both approximations are within a few $\mathrm{kcal} / \mathrm{mol}$ of the exact theory. Once $S_{2}$ is mixed in, this discrepancy disappears. Although surprising, such a result is not in contradiction to theory, since the diagonal approximation is not a guaranteed lower bound to the 1-RDM approximation. However, as expected

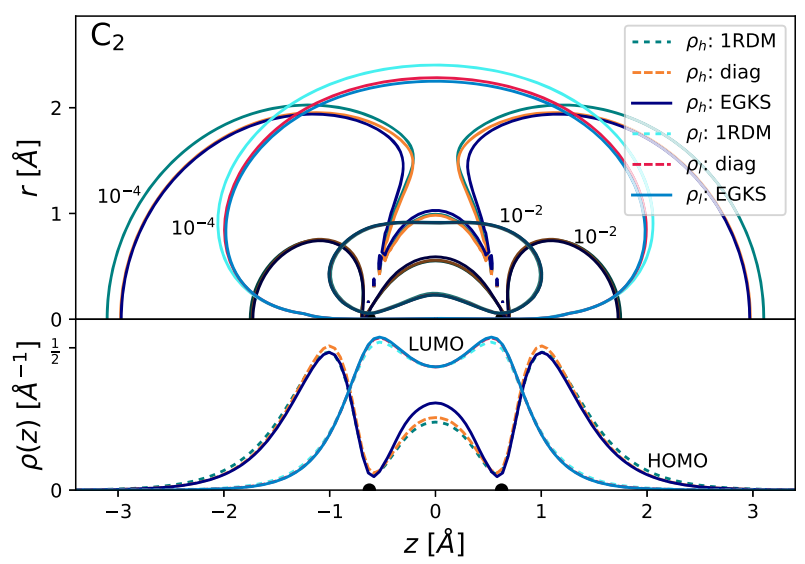

FIG. 4. HOMO and LUMO densities for $\mathrm{C}_{2}$. We retain the colors of previous figures (1-RDM in teal, diag in orange, and EGKS in navy) for the HOMO. LUMO are colored as cyan (1-RDM), red (diag), and blue (EGKS). In the lower panel, we also change the line styles (to dots, dashes, and solid, respectively) to improve clarity. 


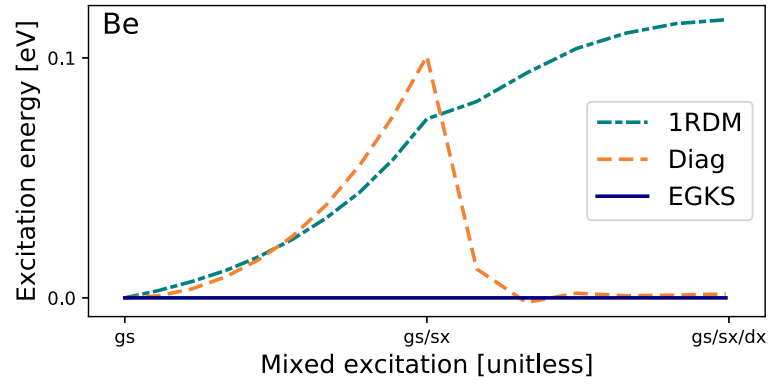

FIG. 5. Energy errors (eV) for singlet excitations of $\mathrm{Be}$. The EGKS error is zero, by definition.

from the improved physics in the diag approximation, this was the only case where it was not a lower bound in practice. We speculate that it might be caused by an increased breaking of spatial symmetry in 1-RDM compared to diag.

\section{CONCLUSIONS}

To conclude, we showed that ensemble KS and generalized KS theories can be unified rigorously into EGKS theory-a good result. However, in doing so directly, one must optimize an energy functional [Eq. (13)] that is not expressible as a 1-RDM functional and, thus, not amenable to a standard GKS treatment-a bad result. We then showed that solutions to EGKS can be defined in its usual operator form [Eq. (16)], provided an orbital-dependent correction term is introduced to the GKS solutions for a small number of "frontier" orbitals that contribute "ghost interactions"-an ugly result. Still, the ghost interaction term can be dealt with via a "diagonal approximation" (Sec. III C) that is reasonably accurate and more amenable to efficient evaluation.

The approach was demonstrated on several analytical and numerical examples based on exchange-only approximations. The diagonal approximation was shown to successfully reproduce exact results (within an exchange-only formalism) and to consistently outperform a simpler 1-RDM approximation, except in one notable case. Our work, thus, demonstrates that not only is ensemble density functional theory formally amenable to a generalized Kohn-Sham framework but also the approach is both practical and advantageous.

Table I and Figs. 1-3 illustrate that both EGKS theory (relative to unrestricted Hartree-Fock) and the diagonal approximation are much better for the two doublet cases than for other cases. We suspect that this reflects the fact that, in doublets, only one electron (h) needs a GI correction, which is, therefore, easier to approximate than the other cases, which require that two electrons ( $h$ and $l$ ) be corrected. The general conditions under which the 1-RDM and/or diag approximations perform well should be scrutinized. Whether successes come from a relative lack of GI errors or a cancellation of GI errors should also be investigated.

It would also be useful to study and develop GKS theory for thermal $\mathrm{EDFT}^{61}$ in both infinite and finite bases. The finite basis case represents a more complex version of the problems discussed here and is amenable to present theory. However, the nature of thermal ensembles means that every excitation (plus promotion/removal of electrons, in the grand canonical case) is included in the ensemble, yielding $F_{i j}^{J} \neq f_{i} f_{j} \forall i, j$. Thus, all orbitals form part of $\mathbb{F}$, and each requires its own GKS equation within the present framework. Whether the 1-RDM and diag approximation work well for such cases should be investigated.

Finally, recent work has shown that any hybrid approximation, $E_{\mathrm{Hxc}}=E_{H}+\alpha E_{\mathrm{x}}+(1-\alpha) E_{\mathrm{x}}^{\mathrm{DFA}}+E_{\mathrm{c}}^{\mathrm{DFA}}$ (or range-separated variants), can be "ensemblized," by setting $\mathcal{E}_{\mathrm{Hxc}}=\mathcal{E}_{\mathrm{H}}+\alpha \mathcal{E}_{\mathrm{x}}+(1-\alpha) \mathcal{E}_{\mathrm{x}}^{\mathrm{DFA}}$ $+\mathcal{E}_{\mathrm{c}}^{\mathrm{DFA} 59}$ and is, thus, amenable to the rigorous EGKS theory introduced here. Here, DFA stands for any standard density functional approximation, and $\mathcal{E}_{\mathrm{x}}^{\mathrm{DFA}} / \mathcal{E}_{\mathrm{c}}^{\mathrm{DFA}}$ are appropriate weighted averages. Furthermore, such functionals can out-perform time-dependent DFT using simplified EGKS schemes. ${ }^{60}$ Logical next steps are, thus, to derive the equivalent of the diagonal approximation for general hybrid theories and to seek better ways of solving the exact theory. This is especially important since most previously evaluated gaps were overestimated, ${ }^{60}$ suggesting that a full or better treatment of EGKS will improve results. Further work is ongoing.

\section{ACKNOWLEDGMENTS}

T.G. and L.K. acknowledge the Australian Research Council (Grant No. DP200100033). T.G. acknowledges support from the National Computational Merit Allocation Scheme (Grant No. ca11). L.K. is the incumbent of the Aryeh and Mintzi Katzmann Chair and acknowledges the Israel Science Foundation.

\section{APPENDIX: PAIR-COEFFICIENTS}

The appendix first derives pair-coefficients for the common ensembles and pure states considered in this work, including which orbitals belong to the double occupied space, $\mathbb{D}$, and the frontier space, $\mathbb{F}$. It then shows how these coefficients combine linearly to produce general ensemble formulae.

\section{Pair-coefficients for singlet ground states}

A singlet ground state represents a pure state,

$$
\hat{\Gamma}_{S_{0}}=\left|S_{0}\right\rangle\left\langle S_{0}|=| 1^{2} 2^{2} \cdots h^{2}\right\rangle\left\langle 1^{2} 2^{2} \cdots h^{2}\right|,
$$

in which all orbitals are doubly occupied or unoccupied. Thus, one trivially finds $f_{i}=2, \forall i \leq h$ and

$$
F_{i j}^{J}=f_{i} f_{j}=4, \quad F_{i j}^{K}=-\frac{1}{2} f_{i} f_{j}=-2,
$$

for the pair-coefficients. These systems, therefore, have no ghost interactions. All occupied orbitals, thus, belong to the doubly occupied space, $\mathbb{D}=\{i \leq h\}$, and the frontier space, $\mathbb{F}=\{\}$, is empty. Any coefficients not specified here, or in the remainder of the appendix, are zero.

\section{Pair-coefficients for doublets}

The next simplest ground state is a doublet, in which all electrons are paired except one, i.e., the state is $\left|1^{2} 2^{2} \cdots h^{\uparrow}\right\rangle$ or $\uparrow \rightarrow \downarrow$. Since our ensemble formalism treats all electrons in the same effective potential, these two states are degenerate (not so in unrestricted Hartree-Fock theory). The relevant ensemble is, thus,

$$
\hat{\Gamma}_{D_{0}}=\frac{1}{2}\left[\left|1^{2} 2^{2} \cdots h^{\uparrow}\right\rangle\left\langle 1^{2} 2^{2} \cdots h^{\uparrow}|+| 1^{2} 2^{2} \cdots h^{\downarrow}\right\rangle\left\langle 1^{2} 2^{2} \cdots h^{\downarrow}\right|\right] .
$$


We may, without any loss of generality, evaluate the density of the $\uparrow$ state to obtain the coefficients $f_{i<h}=2$ and $f_{h}=1$. The HartreeFock energy,

$$
E_{\mathrm{Hx}}^{D_{0}}=\frac{1}{2} \sum_{i, j<h}[4(i j \mid i j)-2(i j \mid j i)]+\frac{1}{2} \sum_{i<h} 2[2(i h \mid i h)-(i h \mid h i)],
$$

then yields the pair-coefficients, which are

$$
F_{i j}^{J}=-2 F_{i j}^{K}= \begin{cases}f_{i} f_{j}, & i<h \text { or } j<h, \\ 0, & i=j=h .\end{cases}
$$

Thus, we see that most terms are in the usual product form, with only the $h h$ term being different. For the purposes of EGKS calculations, $\mathbb{D}=\{i<h\}$ and $\mathbb{F}=\{h\}$, i.e., the doubly occupied orbitals are $i<h$ and the frontier orbital is $h$.

\section{Pair-coefficients for triplets}

An important property of most ensembles (including all we consider here) is that the KS pair-density, and, consequently, its pair-coefficients, must be independent of certain spin-properties of the system. This result follows from the fluctuation-dissipation theorem. ${ }^{59}$ Thus, for the triplet state ensemble,

$$
\hat{\Gamma}_{T_{0}}=\frac{1}{3} \sum_{M_{z} \in\{-1,0,1\}}\left|T_{0, M_{z}}\right\rangle\left\langle T_{0, M_{z}}\right| .
$$

We may pick any of the three triplet states to evaluate the paircoefficients. Taking the $M_{z}=1$ state, $\left|T_{0,1}\right\rangle=\left|1^{2} 2^{2} \cdots h^{\uparrow} l^{\uparrow}\right\rangle$, gives $f_{i<h}$ $=2, f_{h}=f_{l}=1$. The Hartree-Fock energy for this state is

$$
E_{\mathrm{Hx}}^{T_{0}}=\frac{1}{2} \sum_{i j} f_{i} f_{j}(i j \mid i j)-\frac{1}{2} \sum_{i, j \leq l}(i j \mid j i)-\frac{1}{2} \sum_{i, j<h}(i j \mid j i),
$$

where the first term is the Hartree energy and the second and third terms are the exchange of up and down electrons, respectively.

It is readily seen that, for $i, j<h$, we obtain $F_{i j}^{J}=f_{i} f_{j}=-2 F_{i j}^{K}$, a result that also holds for $i h, i l, h i$, and $l i$, provided $i<h$. For the remaining states, we obtain

$$
F_{f g}^{J}=-F_{f g}^{K}=1, \quad \forall f, g \in\{h, l\} .
$$

Thus, we again have only a small number of combinations of $i$ and $j$ for which $F_{i j}^{J} \neq-2 F_{i j}^{K} \neq f_{i} f_{j}$ and, thus, contribute to the ghost interaction term. Thus, $\mathbb{D}=\{i<h\}$ and $\mathbb{F}=\{h, l\}$.

\section{Pair-coefficients for singlet excitations}

In this work, we consider single and double excitations, from $h$ to $l$. The single excitation is represented by a state $\left|S_{1}\right\rangle$ $=\left[\left|1^{2} 2^{2} \cdots h^{\uparrow} l^{\downarrow}\right\rangle+\left|1^{2} 2^{2} \cdots h^{\downarrow} l^{\uparrow}\right\rangle\right] / \sqrt{2}$ and ensemble $\hat{\Gamma}_{S_{1}}=\left|S_{1}\right\rangle\left\langle S_{1}\right|$. Application of the Slater-Condon rules yields

$$
E_{\mathrm{Hx}}^{S_{1}}=E_{\mathrm{Hx}}^{T_{0}}+2(h l \mid l h),
$$

using results for the triplet state. Thus, $F_{i j}^{J}=f_{i} f_{j}=-2 F_{i j}^{K}$, whenever $i<h$ or $j<h$, and

$$
F_{f g}^{J}=F_{f g}^{K}=1-\delta_{f g}, \quad \forall f, g \in\{h, l\} .
$$

Again, $\mathbb{D}=\{i<h\}$ and $\mathbb{F}=\{h, l\}$.
The double excitation is easier to treat, being in the form of a singlet state, $\hat{\Gamma}_{S_{2}}=\left|S_{2}\right\rangle\left\langle S_{2}\right|$ and $\left|S_{2}\right\rangle=\left|1^{2} 2^{2} \cdots h^{0} l^{2}\right\rangle$. Thus, $f_{i}=2$, $\forall i<h, f_{h}=0$, and $f_{l}=1$, and

$$
F_{i j}^{J}=f_{i} f_{j}=4, F_{i j}^{K}=-\frac{1}{2} f_{i} f_{j}=-2,
$$

for the pair-coefficients. Here, $\mathbb{D}=\{i<h, l\}$, and $\mathbb{F}=\{\}$ is empty.

\section{Pair-coefficients for general ensembles}

Finally, we note that the piecewise linearity of ensembles also leads to piecewise linearity of pair-coefficients, provided one starts from an appropriate starting point, like those derived in previous sections.

Thus, for a general ensemble,

$$
\hat{\Gamma}^{\mathrm{w}}=\sum_{\kappa} w_{\kappa} \hat{\Gamma}_{\kappa},
$$

we obtain the general result $f_{i}=\sum_{\kappa} w_{\kappa} f_{i}^{\kappa}$, and

$$
F_{i j}^{J, \mathbf{w}}=\sum_{\kappa} w_{\kappa} F_{i j}^{J, \kappa}, F_{i j}^{K, \mathbf{w}}=\sum_{\kappa} w_{\kappa} F_{i j}^{K, \kappa} .
$$

Importantly, if either $i \in \mathbb{D}$ or $j \in \mathbb{D}$ in all members of the ensemble, then, consequently, $F_{i j}^{J, \mathbf{w}}=f_{i} j_{i}$ and $F_{i j}^{K, \mathbf{w}}=-\frac{1}{2} f_{i} f_{j}$. If, however, both $i \in \mathbb{F}$ and $j \in \mathbb{F}$ (including $i=j$ ) in any member, $\kappa$, then the product form is no longer guaranteed.

For example, the fractional cation, $\mathrm{Li}^{+q}$, is formed as $\hat{\Gamma}=q \hat{\Gamma}_{\mathrm{Li}^{+}}$ $+(1-q) \hat{\Gamma}_{\mathrm{Li}}$, in terms of a singlet cation and doublet neutral atom. We, therefore, obtain $f_{1 s}=2, f_{2 s}=q \times 0+(1-q) \times 1=1-q$, and

$$
\begin{gathered}
F_{1 s 1 s}^{J}=-2 F_{1 s 1 s}^{K}=4, \\
F_{1 s 2 s}^{J}=-2 F_{1 s 2 s}^{K}=2(1-q)=F_{2 s 1 s}^{J}=-2 F_{2 s 1 s}^{K}, \\
F_{2 s 2 s}^{H}=-2 F_{2 s 2 s}^{K}=0 .
\end{gathered}
$$

The fractional anion $\mathrm{Li}^{-q}$ is formed on $\hat{\Gamma}=q \hat{\Gamma}_{\mathrm{Li}^{-}}+(1-q) \hat{\Gamma}_{\mathrm{Li}}$, yielding $f_{1 s}=2, f_{2 s}=q \times 2+(1-q) \times 1=1+q$, and

$$
\begin{gathered}
F_{1 s 1 s}^{J}=-2 F_{1 s 1 s}^{K}=4, \\
F_{1 s 2 s}^{J}=-2 F_{1 s 2 s}^{K}=2(1-q)=F_{2 s 1 s}^{J}=-2 F_{2 s 1 s}^{K}, \\
F_{2 s 2 s}^{J}=q \times 4+(1-q) \times 0=4 q . \\
F_{2 s 2 s}^{K}=q \times-2+(1-q) \times 0=-2 q .
\end{gathered}
$$

In both cases, the $1 s$ is always doubly occupied, so any term involving it has the product form. The $2 s 2 s$ terms do differ from their product form. Thus, both cases have the same doubly occupied (1s) and frontier orbitals $(2 s)$. Sodium would have the same general form, except with more doubly occupied orbitals $\left(1 s, 2 s\right.$, and $\left.2 p_{x, y, z}\right)$ and a different frontier orbital $(3 s)$

For mixtures, $\hat{\Gamma}=(1-w) \hat{\Gamma}_{S_{0}}+w \hat{\Gamma}_{T_{0}}$, or the lowest singlet and triplet state, we can use (A2) and (A8) to obtain $f_{i<h}=2, f_{h}=\frac{3(1-w)}{2}$, and $f_{l}=\frac{1+3 w}{2}$, for the occupation factors. The non-product paircoefficients can be shown to be

$$
F_{f g}^{J}=\left\{\begin{array}{ll}
0, & h h, l l \\
w, & h l, l h,
\end{array} \quad F_{f g}^{K}= \begin{cases}0, & h h, l l \\
-w, & h l, l h .\end{cases}\right.
$$

Here, $\mathbb{D}=\{i<h\}$ and $\mathbb{F}=\{h, l\}$. 
A more complex example is the mixture of $S_{0}, S_{1}$, and $S_{2}$ used for the final figures, with $\hat{\Gamma}=\frac{1-w}{2}\left[\left|S_{0}\right\rangle\left\langle S_{0}|+| S_{1}\right\rangle\left\langle S_{1}\right|\right]+w\left|S_{2}\right\rangle\left\langle S_{2}\right|$. Combining results from Eqs. (A2), (A10), and (A11) gives $f_{i<h}$ $=2, f_{h}=\frac{3(1-w)}{2}$, and $f_{l}=\frac{1+3 w}{2}$, for the occupation factors. The non-product pair-coefficients can be shown to be

$$
F_{f g}^{J}=\left\{\begin{array}{ll}
\frac{5(1-w)}{2}, & h h, \\
\frac{(1+7 w)}{2}, & l l, \\
0, & h l, l h,
\end{array} \quad F_{f g}^{K}= \begin{cases}-(1-w), & h h, \\
-2 w, & l l, \\
\frac{(1-w)}{2}, & h l, l h .\end{cases}\right.
$$

Once again, $\mathbb{D}=\{i<h\}$ and $\mathbb{F}=\{h, l\}$.

\section{DATA AVAILABILITY}

The data that support the findings of this study are available from the corresponding author upon reasonable request.

\section{REFERENCES}

${ }^{1}$ P. Hohenberg and W. Kohn, "Inhomogeneous electron gas," Phys. Rev. 136, B864-B871 (1964).

${ }^{2}$ W. Kohn and L. J. Sham, "Self-consistent equations including exchange and correlation effects," Phys. Rev. 140, A1133-A1138 (1965).

${ }^{3}$ R. G. Parr and W. Yang, Density Functional Theory of Atoms and Molecules (Oxford University Press, New York, 1989).

${ }^{4}$ R. M. Martin, Electronic Structure: Basic Theory and Practical Methods (Cambridge University Press, Cambridge, 2004).

${ }^{5}$ A. D. Becke, "A new mixing of Hartree-Fock and local density-functional theories," J. Chem. Phys. 98, 1372-1377 (1993).

${ }^{6} \mathrm{C}$. Adamo and V. Barone, "Toward reliable density functional methods without adjustable parameters: The PBE0 model," J. Chem. Phys. 110, 6158-6169 (1999).

${ }^{7}$ J. Heyd, G. E. Scuseria, and M. Ernzerhof, "Erratum: 'Hybrid functionals based on a screened Coulomb potential'," J. Chem. Phys. 124, 219906 (2006).

${ }^{8}$ T. Yanai, D. P. Tew, and N. C. Handy, "A new hybrid exchange-correlation functional using the Coulomb-attenuating method (CAM-B3LYP)," Chem. Phys. Lett, 393, 51-57 (2004).

${ }^{9}$ J.-D. Chai and M. Head-Gordon, "Systematic optimization of long-range corrected hybrid density functionals," J. Chem. Phys. 128, 084106 (2008).

${ }^{10} \mathrm{Y}$. Zhao and D. G. Truhlar, "The M06 suite of density functionals for main group thermochemistry, thermochemical kinetics, noncovalent interactions, excited states, and transition elements: Two new functionals and systematic testing of four M06-class functionals and 12 other functionals," Theor. Chem. Acc. 120, 215-241 (2008).

${ }^{11}$ A. Seidl, A. Görling, P. Vogl, J. A. Majewski, and M. Levy, "Generalized KohnSham schemes and the band-gap problem," Phys. Rev. B 53, 3764-3774 (1996).

${ }^{12}$ A. Görling and M. Levy, "Hybrid schemes combining the Hartree-Fock method and density-functional theory: Underlying formalism and properties of correlation functionals," J. Chem. Phys. 106, 2675-2680 (1997).

${ }^{13}$ R. Baer and L. Kronik, “Time-dependent generalized Kohn-Sham theory," Eur. Phys. J. B 91, 170 (2018).

${ }^{14}$ R. Garrick, A. Natan, T. Gould, and L. Kronik, "Exact generalized Kohn-Sham theory for hybrid functionals," Phys. Rev. X 10, 021040 (2020).

${ }^{15}$ J. P. Perdew and K. Schmidt, "Jacob's ladder of density functional approximations for the exchange-correlation energy," AIP Conf. Proc. 577, 1-20 (2001).

${ }^{16}$ R. T. Sharp and G. K. Horton, "A variational approach to the unipotential manyelectron problem," Phys. Rev. 90, 317 (1953).

${ }^{17} \mathrm{~J}$. D. Talman and W. F. Shadwick, "Optimized effective atomic central potential," Phys. Rev. A 14, 36-40 (1976).

${ }^{18}$ T. Grabo, T. Kreibich, and E. K. U. Gross, "Optimized effective potential for atoms and molecules," Mol. Eng. 7, 27-50 (1997).

${ }^{19} \mathrm{E}$. Engel, "Orbital-dependent functionals for the exchange-correlation energy: A third generation of density functionals," in A Primer in Density Functional Theory, edited by C. Fiolhais, F. Nogueira, and M. A. L. Marques (Springer, Berlin, 2003), Chap. 2, pp. 56-122.

${ }^{20}$ S. Kümmel and J. P. Perdew, "Optimized effective potential made simple: Orbital functionals, orbital shifts, and the exact Kohn-Sham exchange potential," Phys. Rev. B 68, 035103 (2003).

${ }^{21}$ S. Kümmel and L. Kronik, "Orbital-dependent density functionals: Theory and applications,” Rev. Mod. Phys. 80, 3-60 (2008).

${ }^{22}$ J. P. Perdew, R. G. Parr, M. Levy, and J. L. Balduz, "Density-functional theory for fractional particle number: Derivative discontinuities of the energy," Phys. Rev. Lett. 49, 1691-1694 (1982).

${ }^{23}$ T. Stein, H. Eisenberg, L. Kronik, and R. Baer, "Fundamental gaps in finite systems from eigenvalues of a generalized Kohn-Sham method," Phys. Rev. Lett. 105, 266802 (2010).

${ }^{24}$ J. P. Perdew, W. Yang, K. Burke, Z. Yang, E. K. U. Gross, M. Scheffler, G. E. Scuseria, T. M. Henderson, I. Y. Zhang, A. Ruzsinszky, H. Peng, J. Sun, E. Trushin, and A. Görling, "Understanding band gaps of solids in generalized Kohn-Sham theory," Proc. Natl. Acad. Sci. U. S. A. 114, 2801-2806 (2017).

${ }^{25}$ T. Stein, L. Kronik, and R. Baer, "Reliable prediction of charge transfer excitations in molecular complexes using time-dependent density functional theory," J. Am. Chem. Soc. 131, 2818-2820 (2009).

${ }^{26} \mathrm{~N}$. T. Maitra, "Charge transfer in time-dependent density functional theory," J. Phys.: Condens. Matter 29, 423001 (2017).

${ }^{27}$ S. Kümmel, "Charge-transfer excitations: A challenge for time-dependent density functional theory that has been met," Adv. Energy Mater. 7, 1700440 (2017).

${ }^{28}$ T. Gould and J. F. Dobson, "The flexible nature of exchange, correlation, and Hartree physics: Resolving 'delocalization' errors in a 'correlation free' density functional," J. Chem. Phys. 138, 014103 (2013).

${ }^{29}$ E. Kraisler and L. Kronik, "Piecewise linearity of approximate density functionals revisited: Implications for Frontier orbital energies," Phys. Rev. Lett. 110, 126403 (2013).

${ }^{30}$ E. Kraisler and L. Kronik, "Fundamental gaps with approximate density functionals: The derivative discontinuity revealed from ensemble considerations," J. Chem. Phys. 140, 18A540 (2014).

${ }^{31} \mathrm{~T}$. Gould and J. Toulouse, "Kohn-Sham potentials in exact density-functional theory at noninteger electron numbers," Phys. Rev. A 90, 050502 (2014).

${ }^{32}$ A. Görling, "Exchange-correlation potentials with proper discontinuities for physically meaningful Kohn-Sham eigenvalues and band structures," Phys. Rev. B 91, 245120 (2015).

${ }^{33}$ B. Senjean and E. Fromager, "Unified formulation of fundamental and optical gap problems in density-functional theory for ensembles," Phys. Rev. A 98, 022513 (2018).

${ }^{34} \mathrm{~B}$. Senjean and E. Fromager, "N-centered ensemble density-functional theory for open systems," Int. J. Quantum Chem. 120, e26190 (2020).

${ }^{35}$ E. K. U. Gross, L. N. Oliveira, and W. Kohn, "Rayleigh-Ritz variational principle for ensembles of fractionally occupied states," Phys. Rev. A 37, 2805-2808 (1988).

${ }^{36}$ E. K. U. Gross, L. N. Oliveira, and W. Kohn, "Density-functional theory for ensembles of fractionally occupied states. I. Basic formalism," Phys. Rev. A 37, 2809-2820 (1988).

${ }^{37}$ L. N. Oliveira, E. K. U. Gross, and W. Kohn, "Density-functional theory for ensembles of fractionally occupied states. II. Application to the he atom," Phys. Rev. A 37, 2821-2833 (1988).

${ }^{38}$ T. Gould and S. Pittalis, "Density-driven correlations in ensemble density functional theory: Insights from simple excitations in atoms," Aust. J. Chem. 73, 714-723 (2020).

${ }^{39}$ M. Filatov and S. Shaik, "A spin-restricted ensemble-referenced Kohn-Sham method and its application to diradicaloid situations," Chem. Phys. Lett. 304, 429-437 (1999).

${ }^{40}$ M. Filatov, M. Huix-Rotllant, and I. Burghardt, "Ensemble density functional theory method correctly describes bond dissociation, excited state electron transfer, and double excitations," J. Chem. Phys. 142, 184104 (2015).

${ }^{41}$ M. Filatov, "Spin-restricted ensemble-referenced Kohn-Sham method: Basic principles and application to strongly correlated ground and excited states of molecules," Wiley Interdiscip. Rev.: Comput. Mol. Sci. 5, 146-167 (2015). 
${ }^{42} \mathrm{M}$. Filatov, "Ensemble DFT approach to excited states of strongly correlated molecular systems," in Density-Functional Methods for Excited States, edited by N. Ferré, M. Filatov, and M. Huix-Rotllant (Springer International Publishing, Cham, 2016), pp. 97-124.

${ }^{43}$ E. Pastorczak, N. I. Gidopoulos, and K. Pernal, "Calculation of electronic excited states of molecules using the Helmholtz free-energy minimum principle," Phys. Rev. A 87, 062501 (2013).

${ }^{44}$ E. Pastorczak and K. Pernal, "Ensemble density variational methods with selfand ghost-interaction-corrected functionals," J. Chem. Phys. 140, 18A514 (2014).

${ }^{45}$ K. Pernal, N. I. Gidopoulos, and E. Pastorczak, "Excitation energies of molecules from ensemble density functional theory," in Advances in Quantum Chemistry (Elsevier, 2016), pp. 199-229.

${ }^{46}$ A. Pribram-Jones, Z.-h. Yang, J. R. Trail, K. Burke, R. J. Needs, and C. A. Ullrich, "Excitations and benchmark ensemble density functional theory for two electrons," J. Chem. Phys. 140, 18A541 (2014).

${ }^{47}$ Z.-h. Yang, J. R. Trail, A. Pribram-Jones, K. Burke, R. J. Needs, and C. A. Ullrich, "Exact and approximate Kohn-Sham potentials in ensemble density-functional theory," Phys. Rev. A 90, 042501 (2014).

${ }^{48}$ Z.-h. Yang, A. Pribram-Jones, K. Burke, and C. A. Ullrich, "Direct extraction of excitation energies from ensemble density-functional theory," Phys. Rev. Lett. 119, 033003 (2017).

${ }^{49} \mathrm{O}$. Franck and E. Fromager, "Generalised adiabatic connection in ensemble density-functional theory for excited states: Example of the $\mathrm{H}_{2}$ molecule," Mol. Phys. 112, 1684-1701 (2014).

${ }^{50}$ K. Deur, L. Mazouin, and E. Fromager, "Exact ensemble density functional theory for excited states in a model system: Investigating the weight dependence of the correlation energy," Phys. Rev. B 95, 035120 (2017).

${ }^{51} \mathrm{~F}$. Sagredo and K. Burke, "Accurate double excitations from ensemble density functional calculations,” J. Chem. Phys. 149, 134103 (2018).

${ }^{52} \mathrm{~K}$. Deur and E. Fromager, "Ground and excited energy levels can be extracted exactly from a single ensemble density-functional theory calculation," J. Chem. Phys. 150, 094106 (2019).

${ }^{53} \mathrm{E}$. Fromager, "Individual correlations in ensemble density-functional theory: State- and density-driven decompositions without additional Kohn-Sham systems," Phys. Rev. Lett. 124, 243001 (2020).

${ }^{54}$ C. Marut, B. Senjean, E. Fromager, and P.-F. Loos, "Weight dependence of local exchange-correlation functionals in ensemble density-functional theory: Double excitations in two-electron systems," Faraday Discuss. 224, 402 (2020).

${ }^{55}$ P.-F. Loos and E. Fromager, "A weight-dependent local correlation densityfunctional approximation for ensembles," J. Chem. Phys. 152, 214101 (2020).

${ }^{56} \mathrm{~T}$. Gould and S. Pittalis, "Hartree and exchange in ensemble density functional theory: Avoiding the nonuniqueness disaster," Phys. Rev. Lett. 119, 243001 (2017).

${ }^{57}$ T. Gould, L. Kronik, and S. Pittalis, "Charge transfer excitations from exact and approximate ensemble Kohn-Sham theory," J. Chem. Phys. 148, 174101 (2018).

${ }^{58} \mathrm{~T}$. Gould and S. Pittalis, "Density-driven correlations in many-electron ensembles: Theory and application for excited states," Phys. Rev. Lett. 123, 016401 (2019).

${ }^{59}$ T. Gould, G. Stefanucci, and S. Pittalis, "Ensemble density functional theory: Insight from the fluctuation-dissipation theorem," Phys. Rev. Lett. 125, 233001 (2020).
${ }^{60} \mathrm{~T}$. Gould, "Approximately self-consistent ensemble density functional theory: Toward inclusion of all correlations," J. Phys. Chem. Lett. 11, 9907-9912 (2020).

${ }^{61}$ N. D. Mermin, "Thermal properties of the inhomogeneous electron gas," Phys. Rev. 137, A1441-A1443 (1965).

${ }^{62}$ S. Pittalis, C. R. Proetto, A. Floris, A. Sanna, C. Bersier, K. Burke, and E. K. U. Gross, "Exact conditions in finite-temperature density-functional theory," Phys. Rev. Lett. 107, 163001 (2011).

${ }^{63}$ We use atomic units, $m=\mathrm{e}^{2} /\left(4 \pi \epsilon_{0}\right)=\hbar=1$, throughout.

${ }^{64} \mathrm{~A}$. Savin and H.-J. Flad, "Density functionals for the Yukawa electron-electron interaction," Int. J. Quantum Chem. 56, 327-332 (1995).

${ }^{65}$ L. Kronik, T. Stein, S. Refaely-Abramson, and R. Baer, "Excitation gaps of finitesized systems from optimally tuned range-separated hybrid functionals," J. Chem. Theory Comput. 8, 1515-1531 (2012).

${ }^{66}$ N. I. Gidopoulos, P. G. Papaconstantinou, and E. K. U. Gross, "Spurious interactions, and their correction, in the ensemble-Kohn-Sham scheme for excited states," Phys. Rev. Lett. 88, 033003 (2002).

${ }^{67}$ The use of a finite Gaussian-type orbital basis means asymptotics are never truly correct. But we expect the asymptotically correct Hamiltonian to yield more accurate orbitals.

${ }^{68}$ T. Gould, S. Pittalis, J. Toulouse, E. Kraisler, and L. Kronik, "Asymptotic behavior of the Hartree-exchange and correlation potentials in ensemble density functional theory," Phys. Chem. Chem. Phys. 21, 19805-19815 (2019).

${ }^{69} \mathrm{~F}$. Weigend and R. Ahlrichs, "Balanced basis sets of split valence, triple zeta valence and quadruple zeta valence quality for $\mathrm{H}$ to $\mathrm{Rn}$ : Design and assessment of accuracy," Phys. Chem. Chem. Phys. 7, 3297 (2005).

${ }^{70}$ R. M. Parrish, L. A. Burns, D. G. A. Smith, A. C. Simmonett, A. E. DePrince, E. G. Hohenstein, U. Bozkaya, A. Y. Sokolov, R. Di Remigio, R. M. Richard, J. F. Gonthier, A. M. James, H. R. McAlexander, A. Kumar, M. Saitow, X. Wang, B. P. Pritchard, P. Verma, H. F. Schaefer, K. Patkowski, R. A. King, E. F. Valeev, F. A. Evangelista, J. M. Turney, T. D. Crawford, and C. D. Sherrill, "Psi4 1.1: An open-source electronic structure program emphasizing automation, advanced libraries, and interoperability," J. Chem. Theory Comput. 13, 3185-3197 (2017).

${ }^{71}$ D. G. A. Smith, L. A. Burns, D. A. Sirianni, D. R. Nascimento, A. Kumar, A. M. James, J. B. Schriber, T. Zhang, B. Zhang, A. S. Abbott, E. J. Berquist, M. H. Lechner, L. A. Cunha, A. G. Heide, J. M. Waldrop, T. Y. Takeshita, A. Alenaizan, D. Neuhauser, R. A. King, A. C. Simmonett, J. M. Turney, H. F. Schaefer, F. A. Evangelista, A. E. DePrince, T. D. Crawford, K. Patkowski, and C. D. Sherrill, "Psi4NumPy: An interactive quantum chemistry programming environment for reference implementations and rapid development," J. Chem. Theory Comput. 14, 3504-3511 (2018)

${ }^{72}$ P. Lykos and G. W. Pratt, "Discussion on the Hartree-Fock approximation," Rev. Mod. Phys. 35, 496-501 (1963).

${ }^{73}$ P. Mori-Sánchez, A. J. Cohen, and W. Yang, "Many-electron self-interaction error in approximate density functionals," J. Chem. Phys. 125, 201102 (2006).

${ }^{74}$ T. Stein, J. Autschbach, N. Govind, L. Kronik, and R. Baer, "Curvature and Frontier orbital energies in density functional theory," J. Phys. Chem. Lett. 3, 3740-3744 (2012). 